\title{
Fractionalizing Majorana Fermions: Non-Abelian Statistics on the Edges of Abelian Quantum Hall States
}

\author{
Netanel H. Lindner \\ Institute of Quantum Information and Matter, California Institute of Technology, Pasadena, California 91125, USA \\ Department of Physics, California Institute of Technology, Pasadena, California 91125, USA \\ Erez Berg \\ Department of Physics, Harvard University, Cambridge, Massachusetts 02138, USA \\ Gil Refael \\ Department of Physics, California Institute of Technology, Pasadena, California 91125, USA
}

Ady Stern

Department of Condensed Matter Physics, Weizmann Institute of Science, Rehovot 76100, Israel

(Received 1 May 2012; published 11 October 2012)

\begin{abstract}
We study the non-Abelian statistics characterizing systems where counterpropagating gapless modes on the edges of fractional quantum Hall states are gapped by proximity coupling to superconductors and ferromagnets. The most transparent example is that of a fractional quantum spin Hall state, in which electrons of one spin direction occupy a fractional quantum Hall state of $\nu=1 / m$, while electrons of the opposite spin occupy a similar state with $\nu=-1 / m$. However, we also propose other examples of such systems, which are easier to realize experimentally. We find that each interface between a region on the edge coupled to a superconductor and a region coupled to a ferromagnet corresponds to a non-Abelian anyon of quantum dimension $\sqrt{2 m}$. We calculate the unitary transformations that are associated with the braiding of these anyons, and we show that they are able to realize a richer set of non-Abelian representations of the braid group than the set realized by non-Abelian anyons based on Majorana fermions. We carry out this calculation both explicitly and by applying general considerations. Finally, we show that topological manipulations with these anyons cannot realize universal quantum computation.

DOI: 10.1103/PhysRevX.2.041002 Subject Areas: Condensed Matter Physics, Superconductivity, Topological Insulators
\end{abstract}

\section{INTRODUCTION}

Recent years have witnessed an extensive search for electronic systems in which excitations ("quasiparticles") follow non-Abelian quantum statistics. In such systems, the presence of quasiparticles, also known as "nonAbelian anyons" [1-5], makes the ground state degenerate. A mutual adiabatic interchange of the positions of the quasiparticles [6] implements a unitary transformation that operates within the subspace of ground states and shifts the system from one ground state to another. Remarkably, this unitary transformation depends only on the topology of the interchange and is insensitive to imprecision and noise. These properties make non-Abelian anyons a testing ground for the idea of topological quantum computation [7]. The search for non-Abelian systems originated from the Moore-Read theory [5] for the $\nu=$ $5 / 2$ fractional quantum Hall $(\mathrm{FQH})$ state and went on to

Published by the American Physical Society under the terms of the Creative Commons Attribution 3.0 License. Further distribution of this work must maintain attribution to the author $(s)$ and the published article's title, journal citation, and DOI. consider other quantum Hall states [8,9], spin systems [10], $p$-wave superconductors [11-13], topological insulators coupled in proximity to superconductors [14,15], and hybrid systems of superconductors coupled to semiconductors where spin-orbit coupling is strong [16-22]. Signatures of Majorana zero modes may have been observed in recent experiments [23-27].

In the realizations based on superconductors, whether directly or by proximity, the non-Abelian statistics results from the occurrence of zero-energy Majorana fermions bound to the cores of vortices or to the ends of onedimensional wires [11-22,28,29]. Majorana-based nonAbelian statistics is, on the theory side, the most solid prediction for the occurrence of non-Abelian statistics, since it is primarily based on the well-tested BCS meanfield theory of superconductivity. Moreover, on the experimental side, it is the easiest realization to observe [23]. The set of unitary transformations that may be carried out on Majorana-based systems is rather limited, however, and does not allow for universal topological quantum computation [30,31].

In this work, we introduce and analyze a non-Abelian system that is based on proximity coupling to a superconductor but goes beyond the Majorana fermion 
paradigm. The system we analyze is based on the proximity coupling of fractional quantum Hall systems or fractional quantum-spin Hall systems [32] to superconductors and ferromagnetic insulators. (We will use the term "fractional topological insulator" [FTI] for a fractional quantum spin Hall system.) The starting point of our approach is the following observation, made by $\mathrm{Fu}$ and Kane [15] when considering the edge states of 2D topological insulators of noninteracting electrons, of which the integer quantum spin Hall state $[33,34]$ is a particular example: In a $2 \mathrm{D}$ topological insulator, the gapless edge modes may be gapped either by breaking time-reversal symmetry or by breaking charge conservation along the edge. The former may be broken by proximity coupling to a ferromagnet (FM), while the latter may be broken by proximity coupling to a superconductor (SC). Remarkably, there must be a single Majorana mode localized at each interface between a region where the edge modes are gapped by a superconductor to a region where the edge modes are gapped by a ferromagnet.

Our focus is on similar situations in cases where the gapless edge modes are of fractional nature. We find that, under these circumstances, the Majorana operators carried by the interfaces in the integer case are replaced by "fractional Majorana operators" whose properties we study.

We consider three types of physical systems. The first [shown schematically in Fig. 1(a)] is that of a 2D fractional topological insulator [32], which may be viewed as a 2D system in which electrons of spin up form an FQH state of a Laughlin [35] fraction $\nu=1 / m$, where $m$ is an odd integer, and electrons of spin down form an $\mathrm{FQH}$ state of a Laughlin fraction $\nu=-1 / m$.

The second system [shown in Fig. 1(b)] is a Laughlin $\mathrm{FQH}$ droplet of $\nu=1 / m$, divided by a thin insulating barrier into an inner disk and an outer annulus. On the (a)

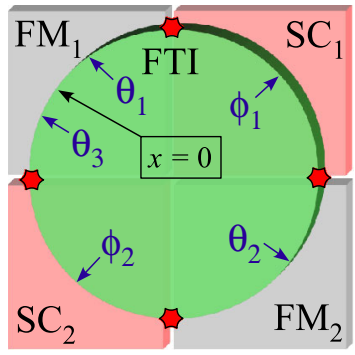

(b)

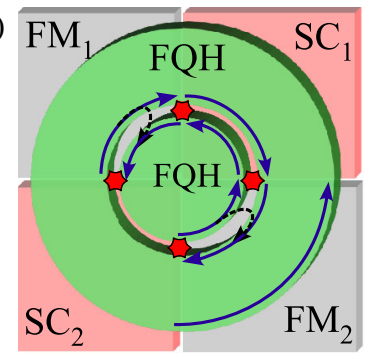

FIG. 1. Schematic setup. (a) A fractional topological insulator (FTI) realization of the system we consider. A FTI droplet with an odd filling factor $1 / \mathrm{m}$ is proximity coupled to ferromagnets (FM) and to superconductors (SC), which gap out its edge modes. The interfaces between the SC and FM segments on the edge of the FTI are marked by red stars. (b) A fractional quantum Hall $(\mathrm{FQH})$ realization of the system we consider. A FQH droplet with filling factor $1 / \mathrm{m}$ is separated by a thin barrier into two pieces: an inner disk and an outer annulus. On either side of the barrier, there are counterpropagating edge states, which are proximity coupled to superconductors and ferromagnets. inner disk, the electronic spins are polarized parallel to the magnetic field (spin up); on the annulus, the electronic spins are polarized antiparallel to the magnetic field (spin down). Consequently, two edge modes flow on the two sides of the barrier, with opposite spins and opposite velocities. Such a state may be created under circumstances where the sign of the $g$ factor is made to vary across the barrier.

The third system is an electron-hole bilayer subjected to a perpendicular magnetic field, in which one layer is tuned to an electron-spin-polarized filling factor of $\nu=1 / \mathrm{m}$, and the other to a hole spin-polarized in the $\nu=-1 / m$ state. In particular, this system may be realized in a material with a spectrum that is electron-hole symmetric, such as graphene.

In all three cases, the gapless edge mode may be gapped by proximity coupling either to a superconductor or to a ferromagnet. We imagine that the edge region is divided into $2 N$ segments, where the superconducting segments are all proximity coupled to the same bulk superconductor, and the ferromagnetic segments are all proximity coupled to the same ferromagnet. The length of each segment is large compared to the microscopic lengths, so that tunneling between neighboring SC-FM interfaces is suppressed. We consider the proximity interactions of the segments with the superconductor and the ferromagnet to be strong.

The questions we ask ourselves are motivated by the analogy with the noninteracting systems of Majorana fermions: What is the degeneracy of the ground state? Is this degeneracy topologically protected? What is the nature of the degenerate ground states? And how can one manipulate the system such that it evolves, in a protected way, between different ground states?

The structure of the paper is as follows: In Sec. II, we give the physical picture that we have developed and summarize our results. In Sec. III, we define the Hamiltonian of the system. In Sec. IV, we calculate the ground-state degeneracy. In Sec. V, we define the operators that are localized at the interfaces and act on the zero-energy subspace. In Sec. VI, we calculate in detail the unitary transformation that corresponds to a braid operation. In Sec. VII, we show how this transformation may be deduced from general considerations, bypassing the need for detailed calculation. In Sec. VIII, we discuss several aspects of the fractionalized Majorana operators and their suitability for topological quantum computation. Section IX contains some concluding remarks. The paper is followed by appendixes that discuss several technical details.

\section{THE PHYSICAL PICTURE AND SUMMARY OF THE RESULTS}

The systems we consider have three types of regions: the bulk, the parts of the edge that are proximity coupled to a superconductor, and the parts of the edge that are proximity coupled to a ferromagnet. 
The bulk is either a fractional quantum Hall state or a fractional quantum spin Hall state. In both cases, the bulk is gapped and incompressible, and its elementary excitations are localized quasiparticles whose charge is a multiple of $e^{*}=e / m$ electron charges. In our analysis, we assume that the area enclosed by the edge modes encloses $n_{\uparrow}$ quasiparticles of spin up and $n_{\downarrow}$ quasiparticles of spin down. These quasiparticles are assumed to be immobile.

In the parts of the edge that are coupled to a superconductor, the charge is defined only modulo $2 e$, because Cooper pairs may be exchanged with the superconductor. Thus, the proper operator to describe the charge on a region of this type is $e^{i \pi \hat{Q}_{i}}$, where $\hat{Q}_{i}$ is the charge in the $i$ th superconducting region. Since the superconducting region may exchange $e^{*}$ charges with the bulk, these operators may take the values $e^{i \pi q_{i} / m}$, with $q_{i}$ an integer whose value is between zero and $2 m-1$. The pairing interaction leads to a ground state that is a spin singlet, and thus the expectation value of the spin within each superconducting region vanishes. As we show below, the Hamiltonian of the system commutes with the operators $e^{i \pi \hat{Q}_{i}}$ in the limit we consider. For the familiar $m=1$ case, these operators measure the parity of the number of electrons within each superconducting region.

The edge regions that are proximity coupled to ferromagnets are, in some sense, the dual of the superconducting regions. The ferromagnet introduces backscattering between the two counterpropagating edge modes, leading to the formation of an energy gap. If the chemical potential lies within this gap, the region becomes insulating and incompressible. Consequently, the charge in the region does not fluctuate, and its value may be defined as zero. The spin, on the other hand, does fluctuate. Since the backscattering from spin-up electron to spin-down electron changes the total spin of the region by two (where the electronic spin is defined as one unit of spin), the operator that may be expected to have an expectation value within the ground state is $e^{i \pi \hat{S}_{i}}$, where $\hat{S}_{i}$ is the total spin in the $i$ th ferromagnet region. Again, spins of $1 / m$ may be exchanged with the bulk, and thus these operators may take the eigenvalues $e^{i \pi s_{i} / m}$, where $s$ is an integer between zero and $2 m-1$. The Hamiltonian of the system commutes with the operators $e^{i \pi \hat{S}_{i}}$ in the limit we consider.

The operators $e^{i \pi \hat{Q}_{i}}$ and $e^{i \pi \hat{S}_{i}}$ label the different domains in the system, as indicated in Fig. 2(a). They satisfy a constraint dictated by the state of the bulk,

$$
\prod_{i=1}^{N} e^{i \pi \hat{Q}_{i}}=e^{i \pi\left(n_{\uparrow}+n_{\downarrow}\right) / m}, \quad \prod_{i=1}^{N} e^{i \pi \hat{S}_{i}}=e^{i \pi\left(n_{\uparrow}-n_{\downarrow}\right) / m} .
$$

For the familiar $m=1$ case, there are only two possible solutions for these constraints, corresponding to the two right-hand sides of Eq. (1) being both +1 or both -1 . For a general $m$, the number of topologically distinct constraints is $2 m^{2}$, since the equations in (1) are invariant

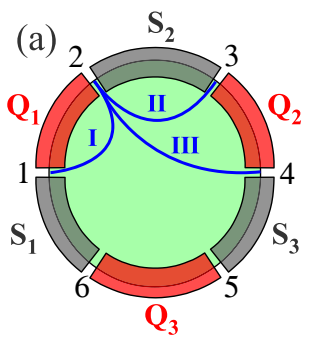

(b)

FIG. 2. Braiding process. (a) A FTI disk with six SC-FM segments. In stages I, II, and III of the braiding process, quasiparticle tunneling (represented by blue curves) is turned on between the SC-FM interfaces. (b) Representation of the braiding procedure, involving interfaces $1,2,3$, and 4 . In the beginning of each stage, the two interfaces connected by a solid line are coupled; during that stage, the bond represented by a dashed line is adiabatically turned on, and, simultaneously, the solid bond is turned off. By the end of stage III, the system returns to the original configuration.

under the transformation where $n_{\uparrow} \rightarrow n_{\uparrow} \pm m$ together with $n_{\downarrow} \rightarrow n_{\downarrow} \pm m$. These sets may be spanned by the values $0 \leq$ $n_{\uparrow} \leq 2 m-1$ and $0 \leq n_{\downarrow} \leq m-1$.

The degeneracy of the ground state may be understood by examining the algebra constructed by the operators $e^{i \pi \hat{Q}_{i}}$ and $e^{i \pi \hat{S}_{i}}$. As we show in the next section, the operators $e^{i \pi \hat{S}_{i}}, e^{i \pi \hat{Q}_{i}}$ satisfy

$$
\begin{aligned}
{\left[e^{i \pi \hat{Q}_{i}}, e^{i \pi \hat{Q}_{j}}\right] } & =\left[e^{i \pi \hat{S}_{i}}, e^{i \pi \hat{S}_{j}}\right]=0, \\
{\left[e^{i \pi \hat{Q}_{j}}, \prod_{i=1}^{N} e^{i \pi \hat{S}_{i}}\right] } & =\left[e^{i \pi \hat{S}_{j}}, \prod_{i=1}^{N} e^{i \pi \hat{Q}_{i}}\right]=0, \\
e^{i \pi \hat{Q}_{j}} e^{i \pi \sum_{k=1}^{l} \hat{S}_{k}} & =e^{i(\pi / m) \delta_{j l}} e^{i \pi \sum_{k=1}^{l} \hat{S}_{k}} e^{i \pi \hat{Q}_{j},}
\end{aligned}
$$

where, in the last equation, $1 \leq j, l<N$. [See Fig. 2(a) for the enumeration convention.] As manifested by Eqs. (2), the pairs of operators $e^{i \pi \hat{Q}_{i}}, e^{i \pi \sum_{k=1}^{i} \hat{S}_{k}}$ form $N-1$ pairs of degrees of freedom, where members of different pairs commute with one another. It is the relation between members of the same pairs, expressed in Eqs. (2), from which the ground-state degeneracy may be easily read out. As is evident from this equation, if $|\psi\rangle$ is a ground state of the system which is also an eigenstate of $e^{i \pi \hat{Q}_{j}}$, then $2 m-1$ additional ground states are $\left(e^{i \pi \sum_{i=1}^{j} \hat{S}_{i}}\right)^{k}|\psi\rangle$, where $k$ is an integer between 1 and $2 m-1$. With $N-1$ mutually independent pairs, we reach the conclusion that the groundstate degeneracy, for a given value of $n_{\uparrow}, n_{\downarrow}$, is $(2 m)^{N-1}$.

The operators acting within a sector of given $n_{\uparrow}, n_{\downarrow}$ of the ground-state subspace are represented by $(2 m)^{N-1} \times$ $(2 m)^{N-1}$ matrices. They may be expressed in terms of sums and products of the operators appearing in Eqs. (2). The physical operations described by the operators $e^{i \pi \hat{S}_{i}}, e^{i \pi \hat{Q}_{i}}$ can also be read off the relations (2). The operator $e^{i \pi \hat{S}_{i}}$ transfers a quasiparticle of charge $e / m$ from the $(i-1)$ th superconductor to the $i$ th superconductor. Since 
the spin within the superconductor vanishes, there is no distinction, within the ground-state manifold, between the possible spin states of the transferred quasiparticle. In contrast, the operator $e^{i \pi \sigma \hat{Q}_{i}}$ transfers a quasiparticle of spin $\sigma= \pm 1$ across the $i$ th superconductor.

For $m=1$, the operators $e^{i \pi \hat{S}_{i}}$ and $e^{i \pi \hat{Q}_{i}}$, measuring the parity of the spin and the charge in the $i$ th ferromagnetic and superconducting region, respectively, may be expressed in terms of Majorana operators that reside at the interfaces bordering that region. A similar representation exists also in the case of $m \neq 1$. Its details are given in Sec. V.

We stress that the ground-state degeneracy is topological, in the sense that no measurement of a local operator can determine the state of the system within the ground-state subspace. For $m=1$, this corresponds to the well-known "topological protection" of the ground-state subspace of Majorana fermions [30,36], as long as single electron tunneling is forbidden either between the different Majorana modes, or between the Majorana modes and the external world. In the fractional case, the states in the ground-state manifold can be labeled by the fractional part of the spin (charge) of the FM (SC) segments, respectively. These clearly cannot be measured locally. Moreover, they can change only by tunneling fractional quasiparticles between different segments; tunneling of electrons from the outside environment cannot split the degeneracy completely, because such tunnelings change the charge and spin of the system only by integers.

Topological manipulations of non-Abelian anyons confined to one dimension are somewhat more complicated than those carried out in two dimensions. The simplest manipulation does not involve any motion of the anyons but rather involves either a $2 \pi$ twist of the order parameter of the superconductor coupled to one or several superconducting segments, or a $2 \pi$ rotation of the direction of the magnetization of the ferromagnet coupled to the insulating segments [37]. When a vortex encircles the $i$ th superconducting region, it leads to the accumulation of a Berry phase of $2 \pi$ multiplied by the number of Cooper pairs it encircles. In the problem we consider, this phase amounts to $e^{i \pi \hat{Q}_{i}}$, and that is the unitary transformation applied by such rotation. As explained above, this transformation transfers a spin of $1 / \mathrm{m}$ between the two ferromagnetic regions that the superconductor borders. Similarly, a rotation of the magnetization in the ferromagnetic region leads to a transfer of a charge of $e / m$ between the two superconductors that the ferromagnet borders.

A more complicated manipulation is that of anyon braiding and its associated non-Abelian statistics. In two dimensions, the braiding of anyons is defined in terms of world lines $\mathbf{R}(t)$ that braid one another as time evolves. On the other hand, in one dimension-both in the integer $m=1$ and in the fractional case-a braiding operation requires the introduction of tunneling terms between different points along the edge [38,39]. The braiding is then defined in terms of trajectories in parameter space, which includes the tunneling amplitudes that are introduced to implement the braiding. The braiding is topological in the sense that it does not depend on the precise details of the trajectory that implements it, as long as the degeneracy of the ground-state manifold does not vary throughout the implementation. Physically, one can imagine realizing such operations by changing external gate potentials that deform the shape of the system's edge adiabatically (similar to the operations proposed for the Majorana case $[38,40])$.

In the integer $m=1$ case, the interchange of two anyons positioned at two neighboring interfaces is carried out by subjecting the system to an adiabatically time-dependent Hamiltonian in which interfaces are coupled to one another. When two or three interfaces are coupled to one another, the degeneracy of the ground state does not depend on the precise value of the couplings, as long as they do not all vanish at once. Consequently, one may "copy" anyon $a$ onto anyon $c$ by starting with a situation where corresponding interfaces $b$ and $c$ are tunnel coupled, and then turning on a coupling between $a$ and $b$ while simultaneously turning off the coupling of $b$ to $c$. Three consecutive "copying" processes then lead to an interchange, and the resulting interchanges generate a non-Abelian representation of the braid group.

In the integer case, only electrons may tunnel between two interfaces, thus allowing us to characterize the tunneling term by one tunneling amplitude. In contrast, in the fractional case, more types of tunneling processes are possible, corresponding to the tunneling of any number of quasiparticles of charges $e / m$ and spin $\pm 1 / m$. To define the effective Hamiltonian coupling two interfaces, we need to specify the amplitudes for all these distinct processes. As one may expect, if only electrons are allowed to tunnel between the interfaces (as may be the case if the tunneling is constrained to take place through the vacuum), the $m=1$ case is reproduced. When single quasiparticles of one spin direction are allowed to tunnel (which is the natural case for the FQHE realization of our model), tunnel coupling between either two or three interfaces reduces the degeneracy of the ground state by a factor of $2 \mathrm{~m}$. This case then opens the way for interchanges of the positions of anyons by the same method envisioned for the integer case. We analyze these interchanges in detail below.

Our analysis of the unitary transformations that correspond to braiding schemes follows two different routes. In the first, detailed in Sec. VI, we explicitly calculate these transformations for a particular case of anyon interchange. In the second, detailed in Sec. VII, we utilize general properties of anyons to all non-Abelian representations of the braid group that satisfy conditions that we impose. It is natural to expect these conditions from the system we analyze. Both routes indeed converge to the same result. While the details of the calculations are given in the following sections, here we discuss their results. 
To consider braiding, we imagine that two anyons at the two ends of the $i$ th superconducting region are interchanged. For the $m=1$ case, the interchange of two Majorana fermions correspond to the transformation

$$
\frac{1}{\sqrt{2}}\left[1 \pm i \exp \left(i \pi \hat{Q}_{i}\right)\right]
$$

This transformation may be written as $\exp \left[i \frac{\pi}{2}\left(\hat{Q}_{i}-k\right)^{2}\right]$, where $k=0,1$ corresponds to the \pm sign in Eq. (3), or as $\frac{1}{\sqrt{2}}\left(1 \pm \gamma_{1} \gamma_{2}\right)$, where $\gamma_{1}, \gamma_{2}$ are the two localized Majorana modes at the two ends of the superconducting region. The square of the transformation is the parity of the charge in the superconducting region. The fourth power of the transformation is unity. Note that, in two dimensions, the two signs in Eq. (3) correspond to anyon exchange in the clockwise and anticlockwise sense. In contrast, in one dimension, the two signs may be realized by different choices of tunneling amplitudes and are not necessarily associated with a geometric notion. Consistent with the topological nature of the transformation, a trajectory that leads to one sign in Eq. (3) cannot be deformed into a trajectory that corresponds to a different sign without passing through a trajectory in which the degeneracy of the ground state varies during the execution of the braiding.

Guided by this familiar example, we expect that, in the fractional case, the unitary transformation corresponding to this interchange will depend only on $e^{i \pi \hat{Q}_{i}}$. We expect to be able to write it as

$$
U\left(\hat{Q}_{i}\right)=\sum_{j=0}^{2 m-1} a_{j} \exp \left(i \pi j \hat{Q}_{i}\right),
$$

with some complex coefficients $a_{j}$, i.e., to be periodic in $\hat{Q}_{i}$, with the period being 2 . We expect the values of $a_{j}$ to depend on the type of tunneling amplitudes that are used to implement the braiding.

In our analysis, we find a more compact, yet equivalent, form for the transformation $U$, which is

$$
U\left(\hat{Q}_{i}\right)=e^{i \alpha \pi\left[\hat{Q}_{i}-(k / m)\right]^{2}} .
$$

The value of $\alpha$ depends on the type of particle that tunnels during the implementation of the braiding, while the value of $k$ depends on the value of the tunneling amplitudes. For an electron tunneling, $\alpha=\frac{m^{2}}{2}$. Just as for the $m=1$ case, for this value of $\alpha$ the unitary transformation (5) has two possible eigenvalues, $U^{4}=1$, and it is periodic in $k$ with a period of 2. For braiding carried out by tunneling single quasiparticles we find $\alpha=\frac{m}{2}$. In this case $U^{4 m}=1$, and $U$ is periodic in $k$ with a period of $2 m$.

Just as in the $m=1$ case, trajectories in parameter space that differ by their value of $k$ are separated by trajectories that involve a variation in the degeneracy of the ground state. We note that, up to an unimportant Abelian phase, the unitary transformation (5) may be thought of as composed of a transformation $e^{i \alpha \pi \hat{Q}_{i}^{2}}$ that results from an interchange of anyons, multiplied by a transformation $e^{(2 \alpha \pi i / m) \hat{Q}_{i} k}$ that results from a vortex encircling the $i$ th superconducting region $2 \alpha \mathrm{k} / \mathrm{m}$ times.

Non-Abelian statistics is the cornerstone of topological quantum computation [7,30], due to the possibility it opens for the implementation of unitary transformations that are topologically protected from decoherence and noise. It is therefore natural to examine whether the non-Abelian anyons that we study allow for universal quantum computation, that is, whether any unitary transformation within the ground-state subspace may be approximated by topological manipulations of the anyons [31]. We find that, at least for unitary time evolution (i.e., processes that do not involve measurements), the answer to this question is negative, as it is for the integer case.

\section{EDGE MODEL}

The edge states of a FTI are described by a hydrodynamic bosonized theory [41,42]. The effective Hamiltonian of the edge is written as

$$
\begin{aligned}
H= & \frac{m u}{2 \pi} \int d x\left[K(x)\left(\partial_{x} \phi\right)^{2}+\frac{1}{K(x)}\left(\partial_{x} \theta\right)^{2}\right] \\
& -\int d x\left[g_{\mathrm{S}}(x) \cos (2 m \phi)+g_{\mathrm{F}}(x) \cos (2 m \theta)\right] .
\end{aligned}
$$

Here, $u$ is the edge-mode velocity; $\phi, \theta$ are bosonic fields satisfying the commutation relation $\left[\phi(x), \theta\left(x^{\prime}\right)\right]=$ $\frac{i \pi}{m} \Theta\left(x^{\prime}-x\right)$, where $\Theta$ is the Heaviside step function; and $g_{\mathrm{S}}(x), g_{\mathrm{F}}(x)$ describe position-dependent proximity couplings to a SC and a FM, which we take to be constant in the SC and FM regions and zero elsewhere, respectively. The magnetization of the FM is taken to be in the $x$ direction. $K(x)$ is a space-dependent Luttinger parameter, originating from interactions between electrons of opposite spins. The charge and spin densities are given by $\rho=\partial_{x} \theta / \pi$ and $s^{z}=\partial_{x} \phi / \pi$, respectively (where the spin is measured in units of the electron spin $\hbar / 2$ ). A right- or left-moving electron is described by the operators $\psi_{ \pm}=e^{i m(\phi \pm \theta)}$.

Crucially for the arguments below, we assume that the entire edge is gapped by the proximity to the SC and FM, except (possibly) the SC-FM interface. This can be achieved, in principle, by making the proximity coupling to the SC and FM sufficiently strong.

\section{GROUND-STATE DEGENERACY OF A DISK WITH $2 N$ SEGMENTS}

We consider a disk with $2 N$ FM-SC interfaces on its boundary [illustrated in Fig. 1(a) for $N=2$ ]. In order to determine the dimension of the ground-state manifold, we construct a set of commuting operators that can be used to characterize the ground states. Consider the operators 
$e^{i \pi Q_{j}} \equiv e^{i\left(\theta_{j+1}-\theta_{j}\right)}, j=1, \ldots, N$, where $\theta_{j}$ is a $\theta$ field evaluated at an arbitrary point near the middle of the $j$ th FM region. The origin $(x=0)$ is chosen to lie within the first FM region [see Fig. 1(a)]. The operator $\theta_{N+1}$ is located within this region, to the left of the origin $(x<0)$, while $\theta_{1}$ is to the right of the origin $(x>0)$. The fields $\theta, \phi$ satisfy the boundary conditions $e^{i \pi Q_{\text {tot }}}=e^{i\left[\theta\left(L^{-}\right)-\theta\left(0^{+}\right)\right]}$and $e^{i \pi S_{\mathrm{tot}}}=e^{i\left[\phi\left(L^{-}\right)-\phi\left(0^{+}\right)\right]}$, where $L$ is the perimeter of the system, and $Q_{\text {tot }}$ and $S_{\text {tot }}$ are the total charge and spin on the edge, respectively.

Since we are in the gapped phase of the sine Gordon model of Eq. (6), we expect in the thermodynamic limit (where the size of all of the segments becomes large) that the $\theta$ field is essentially pinned to the minima of the cosine potential in the FM regions. (Similar considerations hold for the $\phi$ fields in the SC regions.) In other words, the $\theta \rightarrow \theta+\pi / m$ symmetry is spontaneously broken. In this phase, correlations of the fluctuations of $\theta$ decay exponentially on length scales larger than the correlation length $\xi \sim u / \Delta_{\mathrm{F}}$, where $\Delta_{\mathrm{F}}$ is the gap in the FM regions. (See Appendix A for an analysis of the gapped phase.) Therefore, one can construct approximate ground states that are characterized by $\left\langle e^{i\left(\theta_{j+1}-\theta_{j}\right)}\right\rangle=\left\langle e^{i \pi Q_{j}}\right\rangle \equiv \lambda_{j} \neq 0$, where $\lambda_{j}=|\lambda| e^{(i \pi / m) q_{j}}$, and where $q_{j} \in\{0, \ldots, 2 m-1\}$ can be chosen independently for each FM domain. The energy splitting between these ground states is suppressed in the thermodynamic limit as $e^{-R / \xi}$, where $R$ is the length of each region, as discussed below and in Appendix A.

In addition, $e^{i \pi S_{\text {tot }}}$ commutes both with the Hamiltonian and with $e^{i \pi Q_{j}}$. Therefore, the ground states can be chosen to be eigenstates of $e^{i \pi S_{\text {tot }}}$, with eigenvalues $e^{i(\pi / m) s}, s \in$ $\{0, \ldots, 2 m-1\}$. We label the approximate ground states as $|\{q\} ; s\rangle \equiv\left|q_{1}, \ldots, q_{N} ; s\right\rangle$, where $|\{q\} ; s\rangle$ satisfies that $\left\langle\{q\} ; s\left|e^{i \pi Q_{j}}\right|\{q\} ; s\right\rangle=|\lambda| e^{(i \pi / m) q_{j}}$.

For a large but finite system, the $|\{q\} ; s\rangle$ states are not exactly degenerate. There are two effects that lift the degeneracy between them: intrasegment instanton tunneling events between states with different $\{q\}$, and intersegment "Josephson" couplings which make the energy dependent on the values of $\{q\}$. However, both of these effects are suppressed exponentially as $e^{-R / \xi}$, as they are associated with an action that grows linearly with the system size. Therefore, we argue that $|\{q\} ; s\rangle$ are approximately degenerate, up to exponentially small corrections, for any choice of the set $\{q\}, s$.

Similarly, one can define a set of "dual" operators $e^{i \pi S_{j}} \equiv e^{i\left(\phi_{j}-\phi_{j-1}\right)}, \quad j=2, \ldots, N, \quad$ and $\quad e^{i \pi S_{1}}=$ $e^{i \pi S_{\mathrm{tot}}} \prod_{i=2}^{N} e^{-i \pi S_{i}}$. Although the SC regions are in the gapped phase, and the fields $\phi_{j}$ are pinned near the minima of the corresponding cosine potentials, note that the approximate ground states $|\{q\} ; s\rangle$ cannot be further distinguished by the expectation values of the operators $e^{i \pi S_{j}}$. In fact, these states satisfy $\left\langle\{q\} ; s\left|e^{i \pi S_{j}}\right|\{q\} ; s\right\rangle \rightarrow 0$ in the thermodynamic limit because the operators $e^{i \pi S_{j}}$ and $e^{i \pi Q_{j}}$ satisfy the commutation relations

$$
e^{i \pi S_{i}} e^{i \pi Q_{j}}=e^{i \pi / m\left(\delta_{i, j+1}-\delta_{i, j}\right)} e^{i \pi Q_{j}} e^{i \pi S_{i}},
$$

which can be verified by using the commutation relation of the $\phi$ and $\theta$ fields. In the state $|\{q\} ; s\rangle$, the value of $e^{i \pi Q_{j}}$ is approximately localized near $e^{i(\pi / m) q_{j}}$. Applying the operator $e^{i \pi S_{j}}$ to this state shifts $e^{i \pi Q_{j}}$ to $e^{i \pi\left[Q_{j}+(1 / m)\right]}$, as can be seen from Eq. (7). This shift implies that the overlap of the states $|\{q\} ; s\rangle$ and $e^{i \pi S_{j}}|\{q\} ; s\rangle$ decays exponentially with the system size.

Overall, there are $(2 m)^{N+1}$ distinct approximate eigenstate $|\{q\} ; s\rangle$, corresponding to the $2 m$ allowed values of charges $q_{j}$ of each individual SC segment, and the total spin $s$, which can also take $2 m$ values. Not all of these states are physical, however. Labeling the total charge by an integer $q=\sum_{j=1}^{N} q_{j}$, we see from Eq. (1) that $s$ and $q$ must be either both even or both odd, corresponding to a total even or odd number of fractional quasiparticles in the bulk of the system. Because of this constraint, the number of physical states is only $\frac{1}{2}(2 m)^{N+1}$.

In a given sector with a fixed total charge and total spin, there are $N_{\mathrm{GS}}=(2 m)^{N-1}$ ground states. For $m=1$, we obtain $N_{\mathrm{GS}}=2^{N-1}$ for each parity sector, as expected for $2 N$ Majorana states located at each of the FM-SC interfaces [11].

The ground-state degeneracy in the fractional case suggests that each interface can be thought of as an anyon whose quantum dimension is $\sqrt{2 m}$. This is reminiscent of recently proposed models in which dislocations in Abelian topological phases carry anyons with quantum dimensions that are square roots of integers [43-45].

\section{INTERFACE OPERATORS}

We now turn to define physical operators that act on the low-energy subspace. These operators are analogous to the Majorana operators in the $m=1$ case, in the sense that they can be used to express any physical observable in the low-energy subspace. They will be useful when we discuss topological manipulations of the low-energy subspace in the next section.

We define the unitary operators $e^{i \hat{\phi}_{i}}$ and $e^{i \pi \hat{Q}_{j}}$ such that

$$
\begin{array}{r}
e^{i \pi \hat{Q}_{j}}\left|q_{1}, \ldots, q_{N}, s\right\rangle=e^{i \pi q_{j} / m}\left|q_{1}, \ldots, q_{N}, s\right\rangle, \\
e^{i \hat{\phi}_{j}}\left|q_{1}, \ldots, q_{N}, s\right\rangle=\left|q_{1}, \ldots, q_{j}+1, \ldots, q_{N}, s\right\rangle .
\end{array}
$$

$e^{i \pi \hat{Q}_{j}}$ is a diagonal operator in the $|\{q\}, s\rangle$ basis, whereas $e^{i \hat{\phi}_{j}}$ shifts $q_{j}$ by one. These operators can be thought of as projections of the microscopic operators $e^{i \phi_{j}}$ and $e^{i \pi Q_{j}}$, introduced in the previous section, onto the low-energy subspace. In addition, we define the operator $\hat{T}_{s}$ that shifts the total spin of the system:

$$
\hat{T}_{s}\left|q_{1}, \ldots, q_{N}, s\right\rangle=\left|q_{1}, \ldots, q_{N}, s+1\right\rangle .
$$


The operators (9) and (10) will not be directly useful to us, since they cannot be constructed by projecting any combination of edge quasiparticle operators onto the low-energy subspace. To see this, note that they add a charge of $1 / \mathrm{m}$ and zero spin or spin $1 / \mathrm{m}$ with no charge. As a result, they violate the constraint between the total spin and charge, Eq. (1). However, these operators can be used to construct the combinations

$$
\begin{gathered}
\chi_{2 j, \sigma}=e^{i \hat{\phi}_{j}\left(\hat{T}_{s}\right)^{\sigma}} \prod_{i=1}^{j} e^{i \sigma \pi \hat{Q}_{i},} \\
\chi_{2 j+1, \sigma}=e^{i \hat{\phi}_{j+1}\left(\hat{T}_{s}\right)^{\sigma}} \prod_{i=1}^{j} e^{i \sigma \pi \hat{Q}_{i},}
\end{gathered}
$$

where $\sigma= \pm 1$. These combinations, which will be used below, correspond to projections of local quasiparticle operators onto the low-energy manifold. Indeed, the operators $\chi_{j, \sigma}(\sigma= \pm 1)$ carry a charge of $1 / m$ and a spin of $\pm 1 / m$ (as can be verified by their commutation relations with the total charge and total spin operators). Therefore, their quantum numbers are identical to those of a single fractional quasiparticle with spin up or down. Moreover, the commutation relations satisfied by $\chi_{i, \sigma}$ and for $i<j$,

$$
\begin{aligned}
& \chi_{i, \sigma} \chi_{j, \uparrow}=e^{-i(\pi / m)} \chi_{j, \uparrow} \chi_{i, \sigma}, \\
& \chi_{i, \sigma} \chi_{j, \downarrow}=e^{i(\pi / m)} \chi_{j, \downarrow} \chi_{i, \sigma},
\end{aligned}
$$

coincide with those of quasiparticle operators $e^{i \phi(x) \pm i \theta(x)}$ localized at the SC-FM interfaces. (For $i=j,\left[\chi_{j, \uparrow}, \chi_{j, \downarrow}\right]=$ 0 if $j$ is odd, and $\chi_{j, \uparrow} \chi_{j, \downarrow}=e^{2 i \pi / m} \chi_{j, \downarrow} \chi_{j, \uparrow}$ if $j$ is even.) Note that, in our convention, $\chi_{2 j-1, \sigma}$ corresponds to the interface between the segments labeled by $e^{i \pi \hat{S}_{j}}$ and $e^{\pi \hat{Q}_{j}}$, whereas $\chi_{2 j, \sigma}$ corresponds to the interface between $e^{i \pi \hat{Q}_{j}}$ and $e^{i \pi \hat{S}_{j+1}}$; see Fig. 2(a).

Therefore, the operators $\chi_{j, \sigma}$ correspond to quasiparticle creation operators at the SC-FM interfaces, projected onto the low-energy subspace. This conclusion is further supported by calculating directly the matrix elements of the microscopic quasiparticle operator between the approximate ground states, in the limit of strong cosine potentials (see Appendix A). This calculation reveals that the matrix elements of the quasiparticle operators within the lowenergy subspace are proportional to those of $\chi_{j, \sigma}$, and that the proportionality constant decays exponentially with the distance of the quasiparticle operator from the interface. We note that the commutation relations of Eqs. (12) appear in a one-dimensional lattice model of "parafermions" [46,47].

\section{TOPOLOGICAL MANIPULATIONS}

\section{A. Setup}

The braiding process is facilitated by deforming the droplet adiabatically, such that different SC-FM interfaces are brought close to each other at every stage. Proximity between interfaces essentially couples them, by allowing quasiparticles to tunnel between them. We shall assume that only one spin species can tunnel between interfaces. The reason for this assumption will become clear in next sections, and we shall explain how it is manifested in realizations of the model under consideration. At the end of the process, the droplet returns to its original form, but the state of the system does not return to the initial state. The adiabatic evolution corresponds to a unitary matrix acting on the ground-state manifold.

Below, we analyze a braid operation between nearestneighbor interfaces, which we label 3 and 4 (for later convenience). The operation consists of three stages, which are described pictorially in Fig. 2(b). It begins by nucleating a new, small, segment which is flanked by the interfaces 1 and 2. At the beginning of the first stage, the small size of the new segment means that interfaces 1 and 2 are coupled to each other, and all the other interfaces are decoupled. During the first stage, we simultaneously bring interface 3 close to 2 , while moving 1 away from both 2 and 3 , such that at the end of the process only 2 and 3 are coupled to each other, while 1 is decoupled from them. In the second stage, interface 4 approaches 3 , and 2 is taken away from 3 and 4 . In the final stage, we couple 1 to 2 and decouple 4 from 1 and 2, such that the Hamiltonian returns to its initial form. In the following discussion, we analyze an explicit Hamiltonian path yielding this braid operation, which is summarized in Table I. Later, we shall discuss the conditions under which the result is independent of the specific form of the Hamiltonian path representing the same braid operation.

\section{B. Ground-state degeneracy}

To analyze the braiding process, we first need to show that it does not change the ground-state degeneracy. We consider a disk with a total of $N=3$ segments of each type. The ground-state manifold, without any coupling between interfaces, is $(2 m)^{2}$-fold degenerate. We define operators $H_{12}, H_{23}$, and $H_{24}$, the Hamiltonians at the beginning of the three stages I, II, III. These are given by

TABLE I. Summary of the braiding adiabatic trajectory [shown also in Fig. 2(b)]. There are three stages, $\alpha=$ I, II, III, along each of which the parameter $\lambda_{\alpha}$ varies from 0 to 1 . The Hamiltonian in each stage is written in the middle column, where we use the notation $H_{i j}=-t_{i j} \chi_{j, \uparrow} \chi_{i, \uparrow}^{\dagger}+$ H.c. (and where $t_{i j}$ are complex parameters). The right column summarizes the symmetry operators that commute with the Hamiltonian throughout each stage.

\begin{tabular}{lcc}
\hline \hline Stage & Hamiltonian & Symmetries \\
\hline I & $\left(1-\lambda_{\mathrm{I}}\right) H_{12}+\lambda_{\mathrm{I}} H_{23}$ & $e^{i \pi \hat{Q}_{3}}, e^{i \pi \hat{S}_{3}}$ \\
II & $\left(1-\lambda_{\mathrm{II}}\right) H_{23}+\lambda_{\mathrm{II}} H_{24}$ & $e^{i \pi \hat{Q}_{3}}, e^{-i \pi \hat{S}_{1}}$ \\
III & $\left(1-\lambda_{\mathrm{III}}\right) H_{24}+\lambda_{\mathrm{III}} H_{12}$ & $e^{i \pi \hat{Q}_{3}}, e^{-i \pi \hat{Q}_{2}} e^{i \pi \hat{S}_{3}}$ \\
\hline \hline
\end{tabular}




$$
H_{j k}=-t_{j k} \chi_{j, \uparrow} \chi_{k, \uparrow}^{\dagger}+\text { H.c., }
$$

where the $t_{j k}$ are complex amplitudes.

Consider first the initial Hamiltonian (see Table I), given by

$H_{12}=-t_{12} \chi_{2, \uparrow} \chi_{1, \uparrow}^{\dagger}+$ H.c. $=-2\left|t_{12}\right| \cos \left(\pi \hat{Q}_{1}+\varphi_{12}\right)$.

Here, $\varphi_{12}=\arg \left(t_{12}\right)$. It is convenient to work in the basis of eigenstates of the operators $e^{i \pi \hat{Q}_{1}}, e^{i \pi \hat{Q}_{2}}, e^{i \pi \hat{Q}_{3}}$, and $e^{i \pi \hat{S}_{\text {tot }}}$, which we label by $\left|q_{1}, q_{2}, q_{3} ; s\right\rangle$. The total charge and spin are conserved, and we may set $\sum_{j} q_{j}=0$ and $s=0$. Then, a state in the $(2 m)^{2}$-dimensional low-energy subspace can be labeled as $\left|q_{2}, q_{3}\right\rangle$, where $q_{1}$ is fixed to $q_{1}=-q_{2}-q_{3}$. The initial Hamiltonian (14) is diagonal in this basis, and therefore its eigenenergies can be read off easily: $E_{12}\left(q_{2}, q_{3}\right)=-2\left|t_{12}\right| \cos \left[-\frac{\pi}{m}\left(q_{2}+q_{3}\right)+\varphi_{12}\right]$. For generic $\varphi_{12}$, there are $2 m$ ground states. The ground states are nucleated inside a SC region, its total spin is zero, and the ground states are

$$
\left|\Psi_{i}\left(q_{3}\right)\right\rangle=\left|q_{2}=-q_{3}, q_{3}\right\rangle,
$$

labeled by a single index $q_{3}=0, \ldots, 2 m-1$. The residual $2 m$-fold ground-state degeneracy can be understood as a result of the symmetries of the Hamiltonian. From Eq. (14), $e^{i \pi \hat{Q}_{3}}$ and $e^{i \pi \hat{S}_{3}}$ commute with $H_{12}$. The commutation relations between $e^{i \pi \hat{Q}_{3}}$ and $e^{i \pi \hat{S}_{3}}$ ensure that the ground state is (at least) $2 m$-fold degenerate by the eigenvalues of $e^{i \pi \hat{Q}_{3}}$.

Similar considerations can be applied in order to find the ground-state degeneracy throughout the braiding operation. The operator $e^{i \pi \hat{Q}_{3}}$ always commutes with the Hamiltonian, at any stage. This can be seen easily from the fact that the segment labeled by $e^{i \pi \hat{Q}_{3}}$ never couples to any other segment at any stage [see Fig. 2(a)]. Using the definition of the $\chi_{i \sigma}$ operators in Eqs. (11), one finds that

$$
H_{23}=-2\left|t_{23}\right| \cos \left(\pi \hat{S}_{2}+\varphi_{23}\right) \text {, }
$$

and

$$
H_{24}=-2\left|t_{24}\right| \cos \left[\pi\left(\hat{S}_{2}+\hat{Q}_{2}\right)+\varphi_{24}\right] .
$$

In each stage, $\alpha=\mathrm{I}$, II, III, there is a symmetry operator $\Sigma_{\alpha}$ that commutes with the Hamiltonian and satisfies $\Sigma_{\alpha} e^{i \pi \hat{Q}_{3}}=e^{-i(\pi / m)} e^{i \pi \hat{Q}_{3}} \Sigma_{\alpha}$. We specify $\Sigma_{\alpha}$ for each stage in the right column of Table I, and the aforementioned relation can be verified using Eqs. (2). This combination of symmetries dictates that every state is at least $2 m$-fold degenerate, where each degenerate subspace can be labeled by $q_{3}$. Assuming that the special values $\varphi_{12}, \varphi_{23}=$ $\pi(2 l+1) /(2 m)$ and $\varphi_{24}=\pi l / m$ ( $l$ integer) are avoided, the ground state is exactly $2 m$-fold degenerate throughout the braiding process. (The special values for the $\varphi_{i j}$ give an additional twofold degeneracy.) Note that these conclusions hold for any trajectory in Hamiltonian space, as long as the appropriate symmetries are maintained in each stage of the evolution and the accidental degeneracies are avoided.

\section{Braid matrices from Berry's phases}

The evolution operator corresponding to the braid operation can thus be represented as a block-diagonal unitary matrix, in which each $(2 m) \times(2 m)$ block acts on a separate energy subspace. We are now faced with the problem of calculating the evolution operator in the ground-state subspace. Let us denote this operator by $\hat{U}_{34}$, corresponding to a braiding operation of interfaces 3 and 4 . The calculation of $\hat{U}_{34}$ can be done analytically by using the symmetry properties of the Hamiltonian at each stage of the evolution.

We begin by observing that, since $e^{i \pi \hat{Q}_{3}}$ always commutes with the Hamiltonian, $\hat{U}_{34}$ and the evolution operators for each stage are diagonal in the basis of $e^{i \pi \hat{Q}_{3}}$ eigenstates. In every stage, the adiabatic evolution maps $e^{i \pi \hat{Q}_{3}}$ eigenstates between the initial and final ground-state manifolds while preserving the eigenvalue $q_{3}$, and multiplies by a phase factor that may depend on $q_{3}$. This is explicitly summarized as

$$
\hat{U}_{\alpha}\left|\Psi_{i}^{\alpha}\left(q_{3}\right)\right\rangle=\exp \left[i \gamma_{\alpha}\left(q_{3}\right)\right]\left|\Psi_{f}^{\alpha}\left(q_{3}\right)\right\rangle .
$$

Here, $\hat{U}_{\alpha}$ is the evolution operator of stage $\alpha=$ I, II, III, and $\left|\Psi_{i(f)}^{\alpha}\left(q_{3}\right)\right\rangle$ are the ground states of the initial (final) Hamiltonian in stage $\alpha$, respectively, which are labeled by their $e^{i \pi \hat{Q}_{3}}$ eigenvalues. Likewise, $\gamma_{\alpha}\left(q_{3}\right)$ are the phases accumulated in each of the stages.

In order to determine $\gamma_{\alpha}\left(q_{3}\right)$, we use the additional symmetry operator $\Sigma_{\alpha}$ for each stage, as indicated in Table I. This symmetry commutes with the Hamiltonian, and therefore also with the evolution operator for this stage $\left[\Sigma_{\alpha}, \hat{U}_{\alpha}\right]=0$. Acting with $\Sigma_{\alpha}$ on both sides of (18), we find that

$$
\hat{U}_{\alpha} \Sigma_{\alpha}\left|\Psi_{i}^{\alpha}\left(q_{3}\right)\right\rangle=e^{i \gamma_{\alpha}\left(q_{3}\right)} \Sigma_{\alpha}\left|\Psi_{f}^{\alpha}\left(q_{3}\right)\right\rangle .
$$

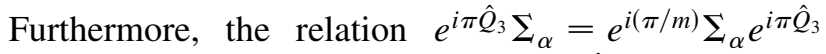
implies that the operator $\Sigma_{\alpha}$ advances $e^{i \pi \hat{Q}_{3}}$ by one increment, and therefore for both the initial and final stage at each stage, we have

$$
\Sigma_{\alpha}\left|\Psi_{i(f)}^{\alpha}\left(q_{3}\right)\right\rangle=\exp \left[i \delta_{i(f)}^{\alpha}\left(q_{3}\right)\right]\left|\Psi_{i(f)}^{\alpha}\left(q_{3}+1\right)\right\rangle,
$$

where $\delta_{i(f)}^{\alpha}\left(q_{3}\right)$ are phases that depend on gauge choices for the different eigenstates, to be determined below. Inserting Eq. (20) into Eq. (19), we obtain the recursion relation

$$
\gamma_{\alpha}\left(q_{3}+1\right)=\gamma_{\alpha}\left(q_{3}\right)+\delta_{f}^{\alpha}\left(q_{3}\right)-\delta_{i}^{\alpha}\left(q_{3}\right) .
$$

Note that, while the phase accumulation at each point along the path depends on gauge choices, the total phase accumulated along a cycle does not. It is convenient to 
choose a continuous gauge, for which the total phases are given by

$$
\hat{U}_{34}\left|\Psi_{i}^{\alpha}\left(q_{3}\right)\right\rangle=\exp \left(i \sum_{\alpha} \gamma_{\alpha}\left(q_{3}\right)\right)\left|\Psi_{i}^{\alpha}\left(q_{3}\right)\right\rangle .
$$

A continuous gauge requires $\left|\Psi_{f}^{\alpha}\left(n_{3}\right)\right\rangle=\left|\Psi_{i}^{\alpha+1}\left(n_{3}\right)\right\rangle$. Therefore, the values of the phases $\delta_{i(f)}^{\alpha}$ depend only on three gauge choices. These are the gauge choices for the eigenstates of the Hamiltonians $H_{12}, H_{23}$, and $H_{24}$, which constitute the initial Hamiltonian at the beginning of stages I through III, as well as the final Hamiltonian for stage III.

Making the necessary gauge choice allows us to solve Eq. (21) for $\gamma_{\alpha}\left(q_{3}\right)$, yielding the total phase (the details of the calculation are given in Appendix B):

$$
\gamma\left(q_{3}\right)=\frac{\pi}{2 m}\left(q_{3}-k\right)^{2}+\gamma_{0}
$$

where $\gamma_{0}$ is an overall ( $q_{3}$-independent) phase that contains both a dynamical phase and a Berry's phase. We cannot compute $\gamma_{0}$ with the present approach, and, moreover, the dynamical phase depends on the details of the path. Note, however, that the differences between the phases of states with different $q_{3}$ are independent of this dynamical phase. In the following discussion, we set $\gamma_{0}=0$ for convenience. The integer $k$ depends on the choice for the phases $\varphi_{i j}$. Recall that the Hamiltonians $H_{i j}$, Eqs. (14), (16), and (17), have an additional degeneracy for a discrete choice of the $\varphi_{i j}$. Any two choices for the $\varphi_{i j}$ that can be deformed to each other without crossing a degeneracy point yield the same $k$.

The evolution operator for the braiding path can be written explicitly by its application on the eigenstates of the Hamiltonian in the beginning of the cycle, $\hat{U}_{34}^{(k)}\left|\Psi_{i}\left(q_{3}\right)\right\rangle=e^{i(\pi / 2 m)\left(q_{3}-k\right)^{2}}\left|\Psi_{i}\left(q_{3}\right)\right\rangle$. Since, by Eq. (15), the ground states of the initial Hamiltonian satisfy $q_{2}=-q_{3}$, the evolution operator can be written in a basis-independent form in terms of the operator $e^{i \pi \hat{Q}_{2}}$. Loosely speaking, $\hat{U}_{34}$ can be written as

$$
\hat{U}_{34}^{(k)}=\exp \left[\frac{i \pi m}{2}\left(\hat{Q}_{2}+\frac{k}{m}\right)^{2}\right] .
$$

Alternatively, using the identity [48] $e^{i(\pi / 2 m) q^{2}}=$ $\sqrt{\frac{1}{2 m}} \sum_{p=0}^{2 m-1} e^{i(\pi / m)\left[p q-\left(p^{2} / 2\right)\right]+i(\pi / 4)}$, one can write

$$
\hat{U}_{34}^{(k)}=\sqrt{\frac{1}{2 m}} \sum_{p=0}^{2 m-1} e^{-(i \pi / 2 m)(p-k)^{2}+i(\pi / 4)}\left(e^{\left.i \pi \hat{Q}_{2}\right)^{p}} .\right.
$$

In the case $m=1, \hat{U}_{34}$ reduces to the braiding rule of Ising anyons [11-13].

Following a similar procedure, one can construct the operator representing the exchange of any pair of neighboring interfaces: $\hat{U}_{2 j-1,2 j}^{(k)}=e^{(i \pi m / 2)\left(\hat{Q}_{j}+k / m\right)^{2}}, \hat{U}_{2 j, 2 j+1}^{(k)}=$ $e^{(i \pi m / 2)\left(\hat{S}_{j+1}+k / m\right)^{2}}$. In order for these operations to form a

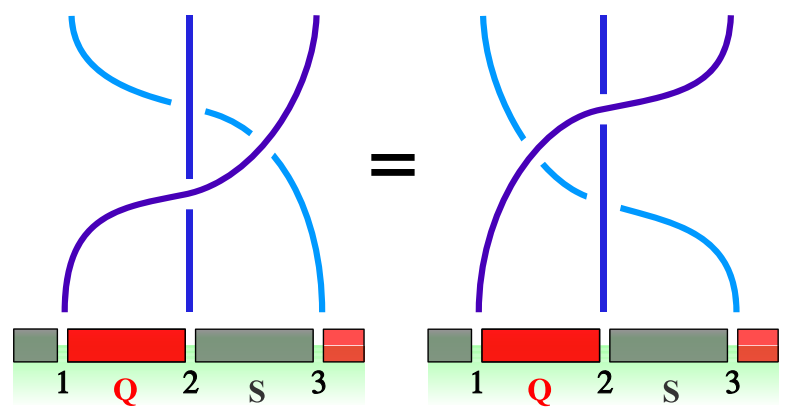

FIG. 3. Diagrammatic representation of the Yang-Baxter equations [Eq. (27)]. Three interfaces 1, 2, 3 are braided in two distinct sequences. The Yang-Baxter equations state that the results of these two sequences of braiding operations are the same.

representation of the braid group, it is necessary and sufficient that they satisfy

$$
\begin{gathered}
{\left[\hat{U}_{i, i+1}^{\left(k_{i}\right)}, \hat{U}_{j, j+1}^{\left(k_{j}\right)}\right]=0 \quad(|i-j|>1),} \\
\hat{U}_{j, j+1}^{\left(k_{1}\right)} \hat{U}_{j+1, j+2}^{\left(k_{2}\right)} \hat{U}_{j, j+1}^{\left(k_{1}\right)}=\hat{U}_{j+1, j+2}^{\left(k_{2}\right)} \hat{U}_{j, j+1}^{\left(k_{1}\right)} \hat{U}_{j+1, j+2}^{\left(k_{2}\right)} .
\end{gathered}
$$

Equation (26) clearly holds because the spin or charge operators of non-nearest-neighbor segments commute. Using Eq. (25), it is not difficult to show that Eq. (27) holds as well. [See Appendix D.] Equation (27) is depicted in Fig. 3. Therefore, $\hat{U}_{i, i+1}$ form a representation of the braid group. For the actual evolution operators (for which the phase $\gamma_{0}$ is path dependent), Eq. (27) can hold only up to an overall phase, and the braiding operations form a projective representation of the braid group. In that respect, our system exhibits a form of non-Abelian statistics. By combining a sequence of nearest-neighbor exchanges, an exchange operation of arbitrarily far interfaces can be defined.

In any physical realization, we do not expect to control the precise form of the Hamiltonian in each stage. It is therefore important to discuss the extent to which the result of the braiding process depends on the details of the Hamiltonian along the path. We argue that the braiding is "topological," in the sense that it is, to a large degree, independent of these precise details.

To see this, one needs to note that the braiding unitary matrix was derived above without referring to the precise adiabatic path in Hamiltonian space. All we use are the symmetry properties of the Hamiltonian in each stage (Table I). These symmetries depend, not on the precise details of the intermediate Hamiltonian, but only on the overall configuration, e.g., which interfaces are allowed to couple in each stage.

In Appendix C 1, we state more formally the conditions under which the result of the braiding is independent of details. Special care must be taken in stage III of the braiding, in which quasiparticles of only one spin species, e.g., spin up, must be allowed to tunnel between interfaces 2 and 4 . We elaborate on the significance of this requirement 
and the ways to meet it in the various physical realizations in Appendix C 2.

\section{BRAIDING AND TOPOLOGICAL SPIN OF BOUNDARY ANYONS}

In the previous section, we derived the unitary matrices representing braid operations by an explicit calculation. In the following discussion, we shall try to shed light on the physical picture behind these representations. To do so, we show that the results of the previous section can be derived almost painlessly, just by assuming that the representation of the braid operations has properties that are analogous to those of anyons in two dimensions. The first and most basic assumption is very natural: There exists a topological operation in the system that corresponds to a braid of two interfaces, in that the unitary matrices representing this operation obey the Yang-Baxter equation[49].

The operations we consider braid two neighboring interfaces, but do not change the total charge (spin) in the segment between them. This results from the general form of the braid operations-To exchange two interfaces flanking a SC (FM) segment, we use couplings to an auxiliary segment of the same type. Therefore, charge (spin) can only be exchanged with the auxiliary segment. Since the auxiliary segment has zero charge (spin) at the beginning and end of the operation, the charge (spin) of the original segment cannot change by the operation. Indeed, this can be seen explicitly in the analysis presented in the previous section. As a result, the unitary matrix representing the braid operation is diagonal with respect to the charge (spin) of the segment flanked by the braided interfaces.

The derivation now proceeds by considering a property of anyons called the topological spin (TS). In two dimensions, the topological spin gives the phase acquired by a $2 \pi$ rotation of an anyon. For fermions and bosons, the topological spin is the familiar -1 and +1 , respectively (corresponding to half-odd or integer spins). There is a close connection between the braid matrix for anyons and their topological spins. In two dimensions, these relations have been considered by various authors $[10,50]$. The system under consideration is one dimensional, and therefore it seemingly does not allow a $2 \pi$ "rotation" of a particle. However, as we shall explain below, the TS of a particle can be defined in our system using the relations of the TS to the braid matrix. We shall then see how to use these relations to derive the possible unitary representations of the braid operations in the system at point.

In our one-dimensional system, we consider the TS of two different kinds of objects (particles): interfaces, which we denote by $X$, and the charge (or spin) of a segment, which we label by $q=0,1, \ldots, 2 m-1$. In what follows, we need to know how to compose or "fuse" different objects in our system. As we saw above, two interfaces yield a quantum number $\exp (i \pi \hat{Q})[\exp (i \pi \hat{S})]$, which is the total charge [spin] in the segment between them, respectively. Suppose we consider two neighboring SC segments with quantum numbers $\exp \left(i \pi \hat{Q}_{1}\right)$ and $\exp \left(i \pi \hat{Q}_{2}\right)$, and we fuse them by shrinking the FM region that lies between them. This fusing results in tunneling of fractional quasiparticles between the two SC regions and energetically favors a specific value for $\exp (i \pi \hat{S})$ in the FM region. The two SC segments are for all purposes one, where clearly, in the absence of other couplings, $\exp \left[i \pi\left(\hat{Q}_{1}+\hat{Q}_{2}\right)\right]$ remains a good quantum number. This therefore suggests the following fusion rules:

$$
\begin{aligned}
X \times X & =0+1+\cdots+2 m-1, \\
q_{1} \times q_{2} & =\left(q_{1}+q_{2}\right) \bmod 2 m .
\end{aligned}
$$

We note that the labeling $q=0,1, \ldots, 2 m-1$ does not depend on the gauge choices in the definition of the operators $\exp (i \pi \hat{Q})$. Equations (28) suggest that the labeling can be defined by the addition law for charges, in which each type of charge plays a different role. Indeed, this addition rule has a measurable physical content that does not depend on any gauge choices.

In two-dimensional theories of anyons, it is convenient to think about particles moving in the two-dimensional plane and to consider topological properties of their world lines (such as braiding). In this paper, we have defined braiding by considering trajectories in Hamiltonian space. In the following discussion, we represent these Hamiltonian trajectories as world lines of the respective "particles" involved, keeping in mind that they do not correspond to motion of objects in real space.

We are now ready to define the TS in our system. In short, the TS of a particle is a phase factor associated with the world line appearing in Fig. 4(a). For interfaces, it is concretely defined by the phase acquired by the system by the following sequence of operations, as illustrated in Fig. 4(e): (i) nucleation of a segment to the right (by convention) of the interface $X_{1}$. (Note that the notation $X_{1}$ corresponds to particle $X$ at coordinate $r_{1}$.) The total spin or charge of this segment is zero (i.e., the nucleation does not add total charge to the system). The couplings between $X_{2}$ and $X_{3}$ flanking the new segment are taken to zero, increasing the ground-state degeneracy by a factor of $2 m$. (ii) A right-handed braid operation is performed between $X_{1}$ and $X_{2}$. (iii) The total charge $q$ of the segment between $X_{2}$ and $X_{3}$ is measured, and we consider (postselect) only the outcomes corresponding to zero charge. Therefore, the system ends up in the same state (i.e., no charges have been changed anywhere in the system), up to a phase factor. Importantly, this phase factor does not depend on the state of the system, since the operation does not change the total charge in the segment of $X_{1}$ and $X_{2}$. (See Appendix E for a more detailed discussion.) We can therefore define this phase factor as $\theta_{X}$, the topological spin of particle type $X$. 
(a)

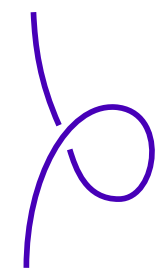

(d)

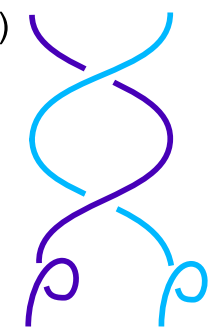

(b)

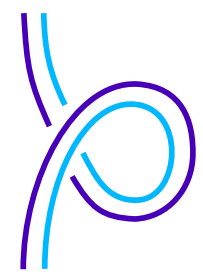

(c)

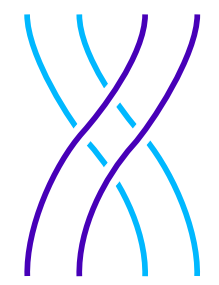

(e)

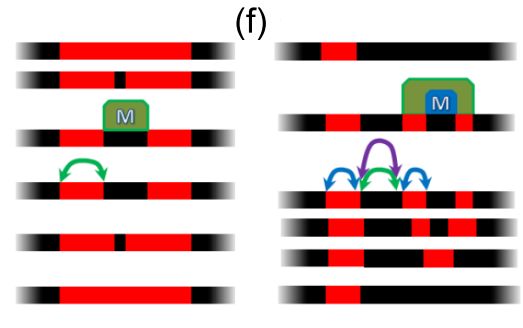

FIG. 4. Topological spin. The process illustrated in (a) defines the TS, where the curves represent the world lines of the $X$ or $q$ particles. A curve forming a cup $\cup$ corresponds to creation of a particle-antiparticle pair (two $X$ 's fusing to $q=0$ or two charges with $q_{1}+q_{2}=0$ ), and a curve forming a cap $\cap$ corresponds to projection on zero total charge. (b) TS of a composite object. When both lines are labeled by $X$ and fuse to charge $q$, the phase acquired is $\theta_{q}$. The fourfold crossing, which is magnified in (c), is assumed to result in an exchange of two $q$ 's. When the lines are labeled by $q_{1}$ and $q_{2}$, the phase acquired is $\theta_{q_{1}+q_{2} \bmod 2 m}$. Importantly, the TS of a composite is equal to the phase accumulated in the process appearing in (d). This equality results from using the Yang-Baxter equation and the definition of the TS. (e) The evolution of the different segments in the process defining $\theta_{X}$. The green arrow corresponds to braiding, while the $\mathrm{M}$ corresponds to projection on zero charge. (f) The process defining $\theta_{q}$. The braids (arrows) are ordered by color: green, blue, and finally purple. The projections are on zero spin for the magnet segment intersecting the two SC segments and zero total charge for these two segments.

In order to define $\theta_{q}$, the topological spin of a charge $q$, we first need to define the operation corresponding to an exchange of two charges. Consider the sequence of four right-handed exchanges of the interfaces, as in Fig. 4(c). The figure suggests that this sequence should yield an exchange of the fusion charges $q_{1}$ and $q_{2}$ of the two pairs of $X$ particles, as would indeed be the case for anyons in two dimensions. (See Appendix E for more details.) Our second assumption is that this is indeed the case. Since by Eqs. (28) there is only one fusion channel for the $q_{i}$ 's, the state is multiplied by a phase factor that depends only on $q_{1}$ and $q_{2}$ - The charges $q_{i}$ are Abelian. It is straightforward to check that exchanges of charges satisfy the Yang-Baxter equation.

The operations defining $\theta_{q}$ are illustrated in Figs. 4(b) and 4(f). We consider, say, a SC segment in an eigenstate of $\exp \left(i \pi \hat{Q}_{1}\right)$ to which we associate a particle type $q_{1}$. In step (i), we nucleate two segments to the right of $q_{1}$. This step is actually performed in two substeps: First, we nucleate a SC segment to the right of the segment $q_{1}$, and then we nucleate a FM segment which separates this segment into two segments, with charges $\exp \left[i \pi\left(\hat{Q}_{2}+\hat{Q}_{3}\right)\right]=1$. Note that the spin of the middle FM segment is also zero, $\exp (i \pi \hat{S})=1$. We now perform a braid between the segments labeled $q_{1}$ and $q_{2}$. Next, we measure the charges $\exp \left[i \pi\left(\hat{Q}_{2}+\hat{Q}_{3}\right)\right]$ and $\exp (i \pi \hat{S})$, postselecting their values to be equal to 1 . The charge $q_{1}$ in the original segment is therefore unchanged by this sequence of operations, as well as any other charges used to label the original state of the system. As before, the state of the system acquires a phase, which depends only on the charge $q_{1}$.

We now use an important relation between the TS of a composite to a double braid of its two components. This relation is a result of an equality between Figs. 4(b) and 4(d), which can be derived by using the Yang-Baxter equation and the definition for the TS. The equality between Figs. 4(b) and 4(d), with both incoming and outgoing lines labeled by $X$, yields

$$
\theta_{q}=\theta_{X}^{2} U^{2}(q)
$$

where $U(q)$ corresponds to the unitary matrix representing an exchange of two interfaces whose fusion charge is $q$. We shall not attempt to calculate $\theta_{X}$. However, by calculating the topological spin $\theta_{q}$ of the composite, we can obtain, using Eq. (29), the square of the sought-after braid matrix, up to a global phase. In fact, in our physical system, this global phase is path dependent.

In two-dimensional theories of anyons, the TS of a composite of two particles is equal to the TS of the particle they fuse into. This relationship can be understood by noting that the equality between Figs. 4(b) and 4(d) guarantees that, in two dimensions, the TS of the composite includes both the intrinsic spin of the two particles and their relative angular momentum. (See Appendix E for more details.) We now assume the same holds in our one-dimensional system-The TS of the $q_{1}, q_{2}$ composite is equal to the TS of combined $q=q_{1}+q_{2} \bmod 2 m$ charge. The TS of a composite of two charges $q_{1}$ and $q_{2}$ is just the process described in Fig. 4(b), with the outgoing lines labeled with $q_{1}$ and $q_{2}$.

Next, we note that, for consistency, the charge $q=0$ must correspond to trivial TS, $\theta_{0}=1$. We take the TS of the elementary charge $q=1$ as a parameter, $\theta_{1}=e^{i \alpha}$, to be determined later. Importantly, note that Fig. 4(a) implies that an exchange of two segments with $q=1$ leads to the phase factor $\theta_{1}$. Using the composition rule in Eqs. (28), $\theta_{q}$ should be equal to the TS of a composite of $q$ unit charges, a process where we encounter $q^{2}$ exchanges of these elementary charges. This gives for the topological spin

$$
\theta_{q \bmod 2 m}=\exp \left(i \alpha q^{2}\right)
$$

The $\mathbb{Z}_{2 m}$ structure above appears since fusing $2 m$ elementary particles results in the trivial $q=0$ charge, and it requires that 


$$
\alpha=\frac{\pi p}{2 m}, \quad p \in \mathbb{Z} .
$$

Taking the square root of Eq. (30), we arrive at

$$
U(q)=(-1)^{f(q)} \exp \left(i \frac{\alpha}{2} q^{2}\right),
$$

where $f(q)$ is an arbitrary, integer-valued function. To determine $\alpha$ and $f(q)$, we appeal to the Yang-Baxter equation, Eq. (27). We find numerically that solutions are possible only for even $p$ 's in Eq. (31), where sign function $(-1)^{f(q)}$ can be of the form $e^{i \pi\left(n_{1} q^{2}+n_{2}\right)}$ with integer $n_{1}, n_{2}$. Therefore, the extra $(-1)$ signs can always be absorbed in the definition of $\alpha$, up to an overall sign. The final result is therefore

$$
U(q)=\exp \left(i \frac{\pi n}{2 m} q^{2}\right), \quad n \in \mathbb{Z} .
$$

As noted in Sec. II, the value $n=1$ is realized by quasiparticle tunnelling of a single spin species, while $n=m$ is realized by electron tunneling. The other representations of the braid group, which are given by $U^{(k)}(q)=$ $\exp \left[i \frac{\pi n}{2 m}(q-k)^{2}\right]$ can be obtained from the above considerations by adding more particles types. Indeed, we see that

$$
U^{(k)}(q)\left[U^{(k-1)}(q)\right]^{\dagger}=e^{-i(\pi n / 2 m)(2 k-1)} e^{i(\pi n / m) q}
$$

is, up to a global phase, a topological operation that is equivalent to taking a vortex $n$ times around a SC segment or changing the orientation of the FM direction $n$ times in the $x-y$ plane by $2 \pi$.

\section{QUANTUM INFORMATION PROCESSING}

In order to assess the suitability of our system for topologically protected quantum computation, it is instructive to examine the structure of the resulting non-Abelian theory. Below, we show that the representation of the braid group realized in our system is a direct product of an Ising anyonic theory times a novel representation of dimension $m$. We argue that braiding operations alone are not sufficient to realize universal topologically protected quantum computation, in agreement with the general argument for models with anyons of quantum dimension which is a square root of an integer [51].

The unitary matrix that describes the braiding operation of two interfaces at the ends of a superconducting segment, Eq. (24), depends on the charge in the superconducting segment. The charge can be written as $Q=q / m$, where $q$ can be uniquely expressed as $q=m q_{\sigma}+2 q_{v}$, with $q_{\sigma}=$ 0,1 and $q_{v}=0, \ldots, m-1$. Inserting this expression into Eq. (24), and assuming for simplicity that $k=0$, we obtain

$$
\hat{U}=e^{(i \pi / 2) q_{\sigma}^{2}} e^{(2 i \pi / m) q_{v}^{2}} .
$$

Writing the Hilbert space $\mathcal{H}$ as a tensor product $\mathcal{H}_{\sigma} \otimes$ $\mathcal{H}_{v}$ such that the states are written as $|q\rangle=\left|q_{\sigma}\right\rangle \otimes\left|q_{v}\right\rangle$ allows us to decompose the braiding matrix into a tensor product $\hat{U}_{\sigma} \otimes \hat{U}_{v}$. Here, $\hat{U}_{\sigma}$ and $\hat{U}_{v}$ are $2 \times 2$ and $m \times m$ matrices, given by the first and second terms on the righthand side of Eq. (35), respectively. A similar decomposition holds for a braid operation acting on a ferromagnetic segment. In this respect, we see that the $2 m$-dimensional representation of the braid group given by Eq. (24) is reducible: It decomposes into a two-dimensional representation, which is nothing but the representation formed by Ising anyons $\sigma$ times an $m$-dimensional representation corresponding to braiding of a non-Ising object $v$.

This decomposition gives insights into the class of unitary transformations that can be realized using braiding of interfaces and hence their suitability for quantum computation. We now argue that, by using braiding operations alone, the system studied in this paper does not allow for topologically protected universal quantum computation. Ising anyons are known not to provide universality for quantum computation $[30,31,52]$. Because of the tensorproduct structure of the topologically protected operations, it is sufficient to consider the fractional part, corresponding to $\mathcal{H}_{v}$. The braid matrices acting within this subspace preserve [53] a generalization of the Pauli group to qudits of dimension $m$. Therefore, the braid operations can be simulated on a classical computer and are not universal.

Conceptually, universality could be achieved by adding an entangling operation between the Ising and the fractional parts. A braiding operation would then produce an effective phase gate that would provide the missing ingredient to make the Ising part universal. However, at present, we do not know whether it is possible to realize such an operation in a topologically protected way. Moreover, topologically protected measurements of charges [54] (and spins) cannot achieve such entanglement, since similarly to the braiding, they can also be shown to be of a tensor-product form.

\section{CONCLUDING REMARKS}

In this work, we have described a physical route for utilizing proximity coupling to superconductors in order to realize a species of non-Abelian anyons, which goes beyond the Majorana fermion paradigm. The essential ingredients of the proposed system are a pair of counterpropagating edge modes of a Laughlin fractional quantum Hall state, proximity coupled to a superconductor. As we have seen, there are several possible realizations of such a system. One could start from a "fractional topological insulator" whose edges are coupled to an array of superconductors and ferromagnets. In the absence of any known realization of a fractional topological-insulator phase (as of today), one could start from an "ordinary" Laughlin fractional quantum Hall state coupled to superconductors. The fractional quantum Hall state in graphene might be a promising candidate for realizing such systems, since the 
magnetic fields needed for observing it are much lower than the fields needed in semiconductor heterostructure devices.

An experimentally accessible signature of the fractionalized Majorana modes is a fractional Josephson effect, which should exhibit a component of $4 m \pi$ periodicity (analogously to the $4 \pi$ periodicity predicted for topological superconductors with Majorana edge modes [15,55]). In addition, it might be possible to observe topological pumping of fractional charge by controlling the relative phase of the superconducting regions.

More broadly speaking, the system we describe here is an example of how gapping out the edge state of a fractionalized two-dimensional phase can realize a topological phase that supports new types of non-Abelian particles, not present in the original two-dimensional theory. In our example, the underlying Laughlin fractional quantum Hall state supports quasiparticles with a fractional charge and fractionalized Abelian statistics; the resulting gapped theory on the edge, however, realizes non-Abelian statistics. Moreover, the resulting non-Abelian theory on the edge is shown to go beyond the well-known Majorana (Ising) framework.

Our results may seem contradictory to the general arguments [56-60] indicating that gapped one-dimensional systems with no symmetry other than fermion parity conservation support only two distinct topological phases, a trivial phase and a nontrivial phase, with an odd number of Majorana modes at the interface between them. The reason our system avoids this exhaustive classification is that it is not, strictly speaking, one dimensional; the edge states of fractional quantum Hall states can never be realized as degrees of freedom of an isolated one-dimensional system. This fact is reflected, for example, in that the theory contains "local" (from the edge perspective) operators that satisfy fractional statistics, which is not possible in any one-dimensional system made of fermions and bosons.

It would be interesting to pursue this idea further by examining gapped states that are realized by gapping out edge modes of topological phases. These states may serve as a route to discovering new classes of topological phases with non-Abelian excitations. For example, more complicated [61] quantum Hall states or higher-dimensional fractional topological insulators [62-64] may be interesting candidates for such investigations.

\section{ACKNOWLEDGMENTS}

N. H. L. and E. B. have contributed equally to this work. We thank Maissam Barkeshli, Lukasz Fidkowsky, Bert Halperin, Alexei Kitaev, Chetan Nayak, and John Preskill for useful discussions. E. B. was supported by the National Science Foundation under Grant Nos. DMR-0757145 and DMR-0705472. A.S. thanks the US-Israel Binational Science Foundation, the Minerva Foundation, and Microsoft Station Q for financial support. N.H.L. and G. R. acknowledge funding provided by the Institute for
Quantum Information and Matter, an NSF Physics Frontiers Center with support of the Gordon and Betty Moore Foundation, and DARPA. N.H.L. was also supported by the David and Lucile Packard Foundation. This work was supported in part by the National Science Foundation Grant No. 1066293 and the hospitality of the Aspen Center for Physics.

Note added.-We recently became aware that a similar idea is being pursued by David Clarke, Jason Alicea, and Kirill Shtengel [65]. In addition, two papers on related subjects $[66,67]$ have appeared.

\section{APPENDIX A: MATRIX ELEMENTS OF THE QUASIPARTICLE OPERATOR}

In this appendix, we describe an explicit calculation of the matrix element of a quasiparticle operator between different states in the ground-state manifold. We show that the matrix element is finite if the quasiparticle is located sufficiently close to an interface between a superconducting and a ferromagnetic segment. The matrix element decays exponentially with the distance from the interface.

\section{Model}

Let us consider a system composed of one superconducting segment, extending from $x=0$ to $x=L$, between two long ferromagnetic segments at $x<0$ and $x>L$. For simplicity, we assume that the gap in the ferromagnetic segments is very large, such that charge fluctuations are completely quenched outside the superconductor. The Hamiltonian for $0 \leq x \leq L$ is

$H=\int_{0}^{L} d x\left\{\frac{m}{2 \pi} u\left[\left(\partial_{x} \phi\right)^{2}+\left(\partial_{x} \theta\right)^{2}\right]-g_{S} \cos (2 m \phi)\right\}$,

supplemented by the boundary condition

$$
\partial_{x} \phi(x=0, L)=0,
$$

which accounts for the fact that the current at the edges of the superconductor is identically zero, due to the large gap in the ferromagnetic regions. We are assuming that the coupling $g_{\mathrm{S}}$ is large enough such that the field $\phi$ is pinned to the vicinity of the minima of the cosine potential, $\phi \approx \frac{\pi}{m} l$, where $l$ is an integer. Deep in the superconducting phase, one can expand the cosine potential up to second order around one of the minima, obtaining the effective Hamiltonian

$$
H_{\mathrm{eff}}=\int_{0}^{L} d x\left\{\frac{m}{2 \pi} u\left[\left(\partial_{x} \phi\right)^{2}+\left(\partial_{x} \theta\right)^{2}\right]+\frac{g}{2}\left(\phi-\frac{\pi l}{m}\right)^{2}\right\},
$$

where $g \equiv(2 m)^{2} g_{S}$.

The Hamiltonian (A3) is quadratic and can be diagonalized using the following mode expansion:

$$
\phi(x)=\frac{\pi}{m} l+\hat{\phi}_{0}+i \frac{1}{\sqrt{m}} \sum_{k=1}^{\infty} \sqrt{\frac{1}{k}} \cos \left[\frac{\pi k}{L} x\right]\left(a_{k}-a_{k}^{\dagger}\right),
$$




$$
\theta(x)=\theta(0)+\frac{\pi \hat{n}}{m L} x+\frac{1}{\sqrt{m}} \sum_{k=1}^{\infty} \sqrt{\frac{1}{k}} \sin \left[\frac{\pi k}{L} x\right]\left(a_{k}+a_{k}^{\dagger}\right)
$$

Here, we have introduced the ladder operators $a_{k}, k=$ $1,2, \ldots$, satisfying $\left[a_{k}, a_{k^{\prime}}^{\dagger}\right]=\delta_{k, k^{\prime}}$ and $\left[a_{k}, a_{k^{\prime}}\right]=0 . \hat{\phi}_{0}$ and $\hat{n}$ are the average phase and the charge of the superconducting segment. These variables are canonical conjugates, satisfying $\left[\hat{\phi}_{0}, \hat{n}\right]=i$. Note that $\left[\theta(0), H_{\mathrm{eff}}\right]=0$; therefore, $\theta(0)$ can be replaced by a c number,

$$
\theta(0)=\frac{\pi}{m} p
$$

with integer $p$, where we have assumed that these values minimize the (infinite) cosine potential on the ferromagnetic side $x<0$. Using Eqs. (A4) and (A5), one can reproduce the commutation relation $\left[\phi(x), \theta\left(x^{\prime}\right)\right]=$ $i \frac{\pi}{m} \Theta\left(x^{\prime}-x\right)$.

Inserting the mode expansions into the Hamiltonian (A3), we obtain

$$
\begin{aligned}
H_{\mathrm{eff}}= & \frac{\pi u}{2 m L} \hat{n}^{2}+\frac{g L}{2} \hat{\phi}_{0}^{2}+\sum_{k=1}^{\infty}\left(\frac{\pi u k}{2 L}+\frac{g L}{4 m k}\right)\left[a_{k}^{\dagger} a_{k}+a_{k} a_{k}^{\dagger}\right] \\
& -\sum_{k=1}^{\infty} \frac{g L}{4 m k}\left(a_{k}^{2}+a_{k}^{\dagger 2}\right) .
\end{aligned}
$$

This Hamiltonian is diagonalized by a Bogoliubov transformation of the form

$$
a_{k}=\alpha_{k} b_{k}+\beta_{k} b_{k}^{\dagger}
$$

where $\alpha_{k}=\sqrt{\frac{1}{2}\left(\frac{A_{k}}{E_{k}}+1\right)}, \quad \beta_{k}=\sqrt{\frac{1}{2}\left(\frac{A_{k}}{E_{k}}-1\right)}, \quad$ and $E_{k}=$ $\sqrt{A_{k}^{2}-B_{k}^{2}}$, expressed via $A_{k}=\frac{\pi u k}{L}+\frac{g L}{2 m k}$ and $B_{k}=\frac{g L}{2 m k}$. The $\hat{n}, \hat{\phi}_{0}$ part of $H_{\text {eff }}$ is diagonalized by introducing ladder operators $\eta, \eta^{\dagger}$ such that

$\hat{\phi}=\left(\frac{g m L^{2}}{\pi u}\right)^{-1 / 4} \frac{\hat{\eta}+\hat{\eta}^{\dagger}}{\sqrt{2}}, \quad \hat{n}=\left(\frac{g m L^{2}}{\pi u}\right)^{1 / 4} \frac{\hat{\eta}-\hat{\eta}^{\dagger}}{i \sqrt{2}}$.

The diagonal form of $H_{\text {eff }}$ (up to constants) is

$$
H_{\mathrm{SC}}=E_{0} \hat{\eta}^{\dagger} \hat{\eta}+\sum_{k=1}^{\infty} E_{k} b_{k}^{\dagger} b_{k},
$$

where $E_{0}=\sqrt{\pi g u / m}$.

\section{Computation of the matrix elements}

Next, we calculate matrix elements of a quasiparticle creation operator between states in the ground-state manifold, which we index by the average values of $\theta$ and $\phi$ on either side of the $x=0$ interface. A diagonal matrix element has the form

$$
A_{l, p}(x)=\left\langle\psi_{l, p}\left|e^{i[\phi(x)+\theta(x)]}\right| \psi_{l, p}\right\rangle,
$$

where $\left|\psi_{l, p}\right\rangle=|\pi l / m, \pi p / m\rangle$ is a ground state in which $\phi(x>0)$ and $\theta(x<0)$ are localized near $\frac{\pi}{m} l$ and $\frac{\pi}{m} p$, respectively. Note that these two variables commute, and therefore they can be localized simultaneously. To evaluate $A_{l, p}(x)$, we use the identity

$$
\left\langle e^{\hat{O}}\right\rangle=e^{\langle\hat{O}\rangle+(1 / 2)\left(\left\langle\hat{O}^{2}\right\rangle-\langle\hat{O}\rangle^{2}\right)},
$$

valid for any operator $\hat{O}$ which is at most linear in creation and annihilation operators. When we substitute $\hat{O}=$ $i[\phi(x)+\theta(x)]$, the expectation values in the exponent can be computed using the mode expansions (A4) and (A5). The computation is lengthy but straightforward, giving

$$
A_{m, n}(x)=e^{i(\pi / m)(l+p)-(1 / 2)\left[F_{\phi}(x)+F_{\theta}(x)\right]},
$$

where

$$
F_{\phi}(x)=\frac{1}{2 L}\left(\frac{m g}{\pi u}\right)^{-1 / 2}+\sum_{k=1}^{\infty} \frac{\pi u}{m L} \frac{\cos ^{2}\left[\frac{\pi k}{L} x\right] e^{-(\alpha \pi k / L)}}{\sqrt{\left(\frac{\pi u k}{L}\right)^{2}+\pi \nu g u}},
$$

$$
\begin{aligned}
F_{\theta}(x)= & \left(\frac{m g}{\pi u}\right)^{1 / 2} \frac{(\pi x / m)^{2}}{2 L} \\
& +\sum_{k=1}^{\infty} \frac{\nu}{k} \frac{\left(\frac{\pi u k}{L}+\frac{g L}{m k}\right) \sin ^{2}\left(\frac{\pi k}{L} x\right) e^{-(\alpha \pi k / L)}}{\sqrt{\left(\frac{\pi u k}{L}\right)^{2}+\frac{\pi}{m} g u}} .
\end{aligned}
$$

Here, we have introduced exponential damping factors of the form $e^{-(\alpha k / L)}$, where $\alpha$ is a short-distance cutoff, to suppress ultraviolet singularities. $F_{\phi}(x)=\left\langle\phi^{2}(x)\right\rangle-$ $\langle\phi(x)\rangle^{2}$, and similarly for $F_{\theta}$. Expectation values of the form $\langle\phi(x) \theta(x)\rangle$ vanish.

We now analyze the asymptotic behavior of $F_{\phi}$ and $F_{\theta}$ for $\xi \ll x \ll L$, where we have defined the correlation length as $\xi=\sqrt{m u /(\pi g)}$. In the limit $L \rightarrow \infty$, the sums over $k$ in Eqs. (A14) and (A15) can be replaced by integrals over $q \equiv k / L$. Then the long-distance asymptotic behavior of $F_{\phi}$ and $F_{\theta}$ is easily extracted:

$$
F_{\phi}(x) \sim \frac{1}{2 m} \ln \left(\frac{\xi}{\alpha}\right)
$$

$F_{\theta}(x) \sim \frac{1}{2 m \xi}\left[-\frac{1}{2} \alpha \ln \frac{\alpha^{2}+4 x^{2}}{\alpha^{2}}+i x \ln \left(\frac{\alpha-2 i x}{\alpha+2 i x}\right)\right]$.

Inserting these expressions into (A13) gives

$$
A_{l, p}(x) \sim e^{i(\pi / m)(l+p)-(\pi / 2 m \xi) x} .
$$

Therefore, the diagonal matrix element of the quasiparticle operator in the ground state $\left|\psi_{l, p}\right\rangle$ decays exponentially with the distance from the interface. In a very similar way, one can show that the matrix element of the quasiparticle operator between two ground states with different $\{l, p\}$ vanishes in the limit $L \rightarrow \infty$. It is therefore natural to identify the operators $\chi_{i, \sigma}$ introduced in Sec. $\mathrm{V}$ as the projection onto the ground-state subspace of the quasiparticle operators $e^{i[\phi(x) \pm \theta(x)]}$ acting at the interface. 


\section{APPENDIX B: CALCULATION OF THE BRAID MATRIX}

In order to complete the calculation of the unitary matrix corresponding to the braiding operation of two interfaces, we first need to obtain the ground states of the Hamiltonians $\mathrm{H}_{12}, \mathrm{H}_{23}$, and $\mathrm{H}_{24}$, making the necessary gauge choices. As in Sec. VI, we use the basis of eigenstates of the operators $\exp \left(i \pi \hat{Q}_{j}\right), j=1,2,3$. We work in the sector $\prod_{j} \exp \left(i \pi \hat{Q}_{j}\right)=1$. A state in this sector can be labeled as $\left|q_{2}, q_{3}\right\rangle$, where $\exp \left(\frac{i \pi}{m} q_{j}\right)$ is the eigenvalue of $\exp \left(i \pi \hat{Q}_{j}\right)$ and $q_{1}=-q_{2}-q_{3}$.

The ground state of $H_{12}=-2\left|t_{12}\right| \cos \left(\pi \hat{Q}_{1}+\varphi_{12}\right)$ is given by

$$
\left|\psi_{i}^{\mathrm{I}}\left(q_{3}\right)\right\rangle=\left|\psi_{f}^{\mathrm{III}}\left(q_{3}\right)\right\rangle=\left|q_{2}=-q_{3}+k_{\mathrm{I}}, q_{3}\right\rangle,
$$

where the integer $k_{\mathrm{I}}$ is determined by $\varphi_{12}$ according to

$$
\frac{\pi}{m}\left(k_{\mathrm{I}}-\frac{1}{2}\right)<\varphi_{12}<\frac{\pi}{m}\left(k_{\mathrm{I}}+\frac{1}{2}\right) .
$$

The ground state is $2 m$-fold degenerate, corresponding to the $2 m$ possible values of $q_{3}$. Equation (B1) includes an explicit gauge choice for the ground states. Note that, for $\varphi_{12}=\frac{\pi}{m}\left(k_{\mathrm{I}}+\frac{1}{2}\right)$, the ground-state degeneracy increases to $4 m$. We therefore assume that these values of $\varphi_{12}$ are avoided.

The Hamiltonian in the beginning of the second stage, $H_{23}$, can be written in the basis of $q_{2}$ eigenstates as

$$
H_{23}=-\left|t_{23}\right| \sum_{q_{2}=0}^{2 m-1} e^{i \varphi_{23}}\left|q_{2}+1\right\rangle\left\langle q_{2}\right|+\text { H.c. }
$$

The above form can be derived from the relation $e^{i \pi \hat{Q}_{2}} e^{i \pi \hat{S}_{2}}=e^{i \pi / m} e^{i \pi \hat{S}_{2}} e^{i \pi \hat{Q}_{2}}$, i.e., $e^{i \pi \hat{S}_{2}}$ is a raising operator for $e^{i \pi \hat{Q}_{2}}$. The Hamiltonian (B3) can be thought of as an effective tight-binding model on a periodic ring of length $2 m$ with complex hopping amplitudes. Note that the total effective flux through the ring is given by

$$
\Phi_{\text {eff }}^{23}=2 m \varphi_{23} .
$$

Importantly, note that when $\Phi_{\text {eff }}^{23}=\pi$, the ground state of $\mathrm{H}_{23}$ is doubly degenerate. These are degeneracy points that we assume are avoided in the braiding process.

The ground state for a particle on a ring with flux $\Phi_{\text {eff }}^{23}$ is simply a plane wave,

$$
\left|\psi_{f}^{\mathrm{I}}\left(q_{3}\right)\right\rangle=\left|\psi_{i}^{\mathrm{II}}\left(q_{3}\right)\right\rangle=\sum_{n=0}^{2 m-1} e^{i(\pi / m) n k_{\mathrm{II}}}\left|q_{2}=-q_{3}+n, q_{3}\right\rangle,
$$

where $k_{\mathrm{II}}$ is the closest integer to $m \varphi_{23} / \pi$. Note that, again, a gauge choice for the overall phase of the states has been made in Eq. (B5).

The phases $\delta_{i, f}^{\mathrm{I}}\left(q_{3}\right)$, defined in Eq. (20) of the main text, are determined by operating with the symmetry operator
$\Sigma_{\mathrm{I}}=\exp \left(i \pi \hat{S}_{3}\right)$ on the ground states of the initial $\left(H_{12}\right)$ or the final $\left(H_{23}\right)$ Hamiltonian, Eqs. (B1) and (B5), respectively. In the gauge we have chosen, this gives

$$
\delta_{f}^{I}\left(q_{3}\right)=\delta_{i}^{I}\left(q_{3}\right)=0
$$

Therefore, the recursion relation for $\gamma_{\alpha}\left(q_{3}\right)$,

$$
\gamma_{\alpha}\left(q_{3}+1\right)=\gamma_{\alpha}\left(q_{3}\right)+\delta_{f}^{\alpha}\left(q_{3}\right)-\delta_{i}^{\alpha}\left(q_{3}\right),
$$

leads to

$$
\gamma_{\mathrm{I}}\left(q_{3}+1\right)=\gamma_{\mathrm{I}}\left(q_{3}\right)
$$

Since we are not interested in the overall ( $q_{3}$-independent) phase of the braid matrix, we can set, for stage I, $\gamma_{\mathrm{I}}\left(q_{3}\right)=0$, which satisfies Eq. (B8).

The Hamiltonian at the end of stage II, $H_{24}$, can be written as

$$
\begin{aligned}
H_{24} & =-\left|t_{24}\right| e^{i \varphi_{24}} \chi_{4 \uparrow} \chi_{2 \uparrow}^{\dagger}+\text { H.c. } \\
& =-\left|t_{24}\right| e^{i \varphi_{24}} e^{i \pi \hat{S}_{2}} e^{i \pi \hat{Q}_{2}}+\text { H.c. }
\end{aligned}
$$

In the second line, we have used the explicit form of $\chi_{2,4 \uparrow}$ in terms of the spin and charge operators. Writing the Hamiltonian in the basis of eigenstates of $e^{i \pi \hat{Q}_{2}}$, we obtain

$H_{24}=-\left|t_{24}\right| \sum_{q_{2}=0}^{2 m-1} e^{i \varphi_{24}+i(\pi / m) q_{2}}\left|q_{2}+1\right\rangle\left\langle q_{2}\right|+$ H.c.

In order to diagonalize $H_{24}$, we perform a gauge transformation to a new basis $\left|\xi_{q_{2}}\right\rangle$ defined as

$$
\left|q_{2}\right\rangle=e^{-i(\pi / 2 m) q_{2}^{2}}\left|\xi_{q_{2}}\right\rangle .
$$

This transformation is designed such that, in the new basis, the phases of the hopping amplitudes are uniform. The Hamiltonian takes the form

$H_{24}=-\left|t_{24}\right| \sum_{q_{2}=0}^{2 m} e^{i\left[\varphi_{24}-(\pi / 2 m)\right]}\left|\xi_{q_{2}+1}\right\rangle\left\langle\xi_{q_{2}}\right|+$ H.c.

which is easily diagonalized in the basis of plane waves. One can verify that, defining $\left|\tilde{\xi}_{p}\right\rangle=\frac{1}{\sqrt{2 m}} \times$ $\sum_{q_{2}=0}^{2 m-1} e^{i(\pi / m) p q_{2}}\left|\xi_{q_{2}}\right\rangle$

$H_{24}\left|\tilde{\xi}_{p}\right\rangle=-2\left|t_{24}\right| \cos \left[\frac{\pi}{m}\left(p+\frac{1}{2}\right)-\varphi_{24}\right]\left|\tilde{\xi}_{p}\right\rangle$.

Therefore, for $\frac{\pi}{m} k_{\mathrm{III}}<\varphi_{24}<\frac{\pi}{m}\left(k_{\mathrm{III}}+1\right)$ where $k_{\mathrm{III}}$ is an integer, we find that the ground state occurs for $p=$ $k_{\mathrm{III}} \bmod (2 m)$. Using Eq. (B11), one can express the ground state in terms of the original basis states: 


$$
\begin{aligned}
\left|\psi_{f}^{\mathrm{II}}\left(q_{3}\right)\right\rangle & =\left|\psi_{i}^{\mathrm{III}}\left(q_{3}\right)\right\rangle \\
& =\frac{e^{-i(\pi / 2 m) k_{\mathrm{III}}^{2}}}{\sqrt{2 m}} \sum_{q_{2}=0}^{2 m-1} e^{i(\pi / 2 m)\left(q_{2}+k_{\mathrm{III}}\right)^{2}}\left|q_{2}, q_{3}\right\rangle
\end{aligned}
$$

Applying the symmetry operator $\Sigma_{\mathrm{II}}=e^{-i \pi \hat{S}_{1}}$ to both sides of (B1) and (B14), we obtain

$$
\delta_{i}^{\mathrm{II}}\left(q_{3}\right)=-\frac{\pi}{m} k_{\mathrm{II}}, \quad \delta_{f}^{\mathrm{II}}\left(q_{3}\right)=0 .
$$

Therefore, solving the recursion relation [Eq. (B7)] and choosing a gauge such that $\gamma_{\mathrm{II}}\left(q_{3}=0\right)=0$, we obtain

$$
\gamma_{\mathrm{II}}\left(q_{3}\right)=\frac{\pi}{m} k_{\mathrm{II}} q_{3} \text {. }
$$

For the last stage of the evolution, applying $\Sigma_{\text {III }}=$ $e^{-i \pi \hat{Q}_{2}} e^{i \pi \hat{S}_{3}}$ to both sides of Eqs. (B14) and (B1), we obtain

$\delta_{i}^{\mathrm{III}}\left(q_{3}\right)=\frac{\pi}{m}\left(k_{\mathrm{III}}+\frac{1}{2}\right), \quad \delta_{f}^{\mathrm{III}}\left(q_{3}\right)=\frac{\pi}{m}\left(q_{3}-k_{\mathrm{I}}+1\right)$.

Inserting this result into Eq. (B7) and solving for $\gamma_{\mathrm{III}}\left(q_{3}\right)$, we obtain

$$
\gamma_{\mathrm{III}}\left(q_{3}\right)=\frac{\pi}{2 m}\left(q_{3}-k_{\mathrm{I}}-k_{\mathrm{III}}\right)^{2} .
$$

The total phase $\gamma=\gamma_{\mathrm{I}}+\gamma_{\mathrm{II}}+\gamma_{\mathrm{III}}$, up to a $q_{3}$-independent phase, is

$$
\gamma\left(q_{3}\right)=\frac{\pi}{2 m}\left(q_{3}-k\right)^{2} .
$$

Here, $k=k_{\mathrm{I}}-k_{\mathrm{II}}+k_{\mathrm{III}}$. Note that, while $\gamma_{\alpha}$ depend on our various gauge choices for the bases of the eigenstates of $H_{12}, H_{23}$, and $H_{24}$, the phase accumulated along the entire path [Eq. (B19)] does not depend on these gauge choices.

\section{APPENDIX C: TOPOLOGICAL PROTECTION OF THE BRAID OPERATIONS}

\section{Independence of microscopic details}

In any physical realization, one would not be able to control the precise form of the Hamiltonian in each stage of the braid process. It is therefore important to discuss to what extent the result of the braiding depends on the details of the Hamiltonian along the path. Below, we argue that the braiding is topological, in the sense that it is independent of these precise details.

Let us begin by noting that the evolution operator describing the full braiding process depends only on the following:

(1) The initial and final Hamiltonians at each stage;

(2) The symmetries of the Hamiltonian at each stage;

(3) The fact that the ground-state degeneracy throughout the process is fixed, such that the evolution can be considered adiabatic.

One can see that the precise details of the timedependent Hamiltonian during the braiding process are unimportant for our derivation of the evolution operator in Sec. VI. Note that we have never used the exact form of the Hamiltonian during the path to determine the evolution operator.

In order to make this argument more formal, let us define $P_{1}$ as the closed path in Hamiltonian space, $H_{12} \rightarrow H_{23} \rightarrow$ $H_{24} \rightarrow H_{12}$, for which we computed the evolution operator. ( $P_{1}$ is summarized in Table I.) Suppose that we replace $P_{1}$ by a different, "realistic" path $P_{2}$, defined as $\tilde{H}_{12} \rightarrow$ $\tilde{H}_{23} \rightarrow \tilde{H}_{24} \rightarrow \tilde{H}_{12}$, which has the same symmetries as those of the original trajectory in each stage (Table I). $P_{1}$ and $P_{2}$ are represented in Figs. 5(a) and 5(b), respectively. We assume further that the Hamiltonian at the end of every stage of $P_{2}$ is adiabatically connectable to that of the original path $P_{1}$, e.g., $\tilde{H}_{12}$ and $H_{12}$ are adiabatically connectable, etc. We argue that the adiabatic evolution associated with $P_{2}$ is unitarily equivalent to that of $P_{1}$. To show this, consider the modified path $P_{3}$ shown in Fig. 5:

$$
\begin{aligned}
H_{12} & \rightarrow \tilde{H}_{12} \rightarrow \tilde{H}_{23} \rightarrow H_{23} \rightarrow \tilde{H}_{23} \rightarrow \tilde{H}_{24} \\
& \rightarrow H_{24} \rightarrow \tilde{H}_{24} \rightarrow \tilde{H}_{12} \rightarrow H_{12} .
\end{aligned}
$$

Clearly, (C1) can be viewed as a deformed version of the original trajectory $P_{1}$, in which the intermediate Hamiltonian during each stage is deformed relative to the original trajectory of Table I. Since the intermediate Hamiltonian in every stage of trajectory (C1) has the same symmetries as those of the original trajectory, the analysis outlined in the previous section shows that the evolution operator representing the overall trajectory (C1) is $e^{i \omega} \hat{U}_{34}$, where $\omega$ is a global phase factor.

On the other hand, we can consider $P_{3}$ starting and ending with $\tilde{H}_{12}$ as the realistic trajectory $P_{2}$. Since, for example, the step $\tilde{H}_{23} \rightarrow H_{23}$ is "undone" by the next step $H_{23} \rightarrow \tilde{H}_{23}, P_{2}$ is unitarily related to $P_{3}$ by $e^{i \omega} V \hat{U}_{34} V^{\dagger}$,

(a)

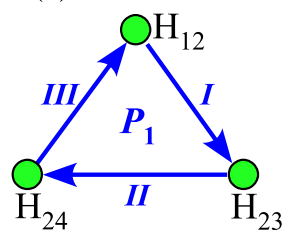

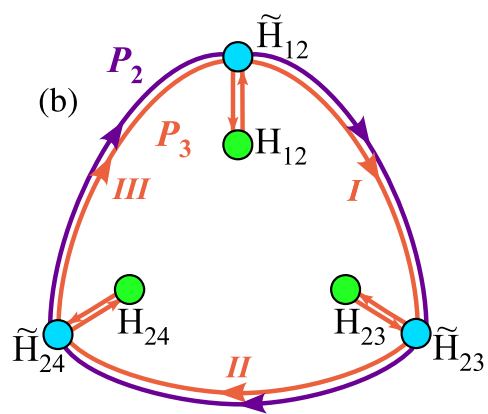

FIG. 5. Braiding paths in Hamiltonian space. (a) Path $P_{1}$, for which we compute the braiding adiabatic evolution operator explicitly. (b) A different path $P_{2}$ (blue), whose Hamiltonians at the intermediate stages are assumed to be adiabatically connectable to those of $P_{1} . P_{3}$ (red) is a path equivalent to $P_{2}$, in which each intermediate Hamiltonian of $P_{2}$ evolves to the corresponding Hamiltonian of $P_{1}$ and then back. 
where $V$ represents the evolution from $\tilde{H}_{12}$ to $H_{12}$. In essence, the matrix $V$ relates the eigenstates of the realistic initial Hamiltonian $\tilde{H}_{12}$ to those of $H_{12}$. We conclude that the adiabatic evolutions corresponding to paths $P_{1}$ and $P_{2}$ are physically equivalent, and therefore the braiding operation is robust to changes in the path in Hamiltonian space, as long as the conditions (1)-(3) listed above are met.

\section{Symmetries of the Hamiltonian during the braiding process}

Next, we discuss the symmetry requirements in every stage in more physical terms. The topological stability of the braiding operation depends crucially on the symmetries of the Hamiltonian throughout the different stages of the braiding operation. We now argue that these symmetry properties are largely independent of the microscopic details of the Hamiltonian in each stage. This is true since the definition of the braid operation contains only information regarding which interfaces are brought in proximity at each stage. For instance, any Hamiltonian trajectory corresponding to this braid operation couples only interfaces 1, 2, and 3 during stage I (see Fig. 2). Any such Hamiltonian necessarily commutes with $e^{i \pi \hat{S}_{3}}$ and $e^{i \pi \hat{Q}_{3}}$, independent of its microscopic details. For example, adding terms such as higher powers of $\chi_{2, \uparrow} \chi_{1, \uparrow}^{\dagger}$ retains these symmetries. Likewise, terms representing direct coupling between interfaces 1 and 3, such as powers of $\chi_{3, \uparrow} \chi_{1, \uparrow}^{\dagger}$, can be added, as long as they are absent at the beginning and end of stage I, when interfaces 1 and 3 are far apart.

A similar statement can be made for stage II: As long as interfaces 5, 6, and 1 remain decoupled throughout the evolution, the Hamiltonian necessarily maintains the same symmetries as those in Table I, regardless of the microscopic details of the process.

The symmetry requirement in stage III requires more care. At this stage, interfaces 1, 2, and 4 are coupled. Crucially, we note that the commutation relations in Eqs. (12) give $\left[\chi_{i \downarrow}, \chi_{j \uparrow} \chi_{k \uparrow}^{\dagger}\right]=0$ for any $j, k \neq i$. Therefore, as long as we allow tunneling of only spin-up particles between interfaces 1,2 , and 4 , we are assured that $\chi_{3, \downarrow}$ commutes with the Hamiltonian. It follows that $\chi_{5, \downarrow} \chi_{3, \downarrow}^{\dagger}=e^{-i \pi \hat{Q}_{2}} e^{i \pi \hat{S}_{3}}$, the symmetry operator required in stage III, also commutes with the Hamiltonian. Again, this symmetry would be maintained independent of the exact form of the Hamiltonian, as long as it obeys the above restriction. The physical reason behind this symmetry is clarified by noting that transferring $n$ up-spin quasiparticles to interface 4 (from either 1 or 2) changes $e^{i \pi \hat{Q}_{2}} \rightarrow$ $e^{i \pi\left[\hat{Q}_{2}+(n / m)\right]}$ and $e^{i \pi \hat{S}_{3}} \rightarrow e^{i \pi\left[\hat{S}_{3}+(n / m)\right]}$, leaving $e^{-i \pi \hat{Q}_{2}} e^{i \pi \hat{S}_{3}}$ invariant.

We now see why it is crucial, in order to allow for the braiding operation, to have only one species of quasiparticles tunnelling between interfaces. If quasiparticles of both spins are allowed to tunnel, $e^{-i \pi \hat{Q}_{2}} e^{i \pi \hat{S}_{3}}$ would cease to be a good symmetry-In fact, the symmetry of the Hamiltonian is lowered, and the ground-state degeneracy is reduced from $(2 \mathrm{~m})$ to just 2 , violating the adiabaticity of the braiding process. This is a special property of the fractional $(m>1)$ case; for $m=1$, there is no difference between up and down quasiparticle tunneling.

We note that the restriction to single-species tunneling was unnecessary in stages I and II, which retain the same symmetries even when both spin species are allowed to tunnel. Moreover, if we allowed only spin-down quasiparticles to tunnel between 2 and 4 , there would be an alternative symmetry operator $e^{i \pi \hat{Q}_{2}} e^{i \pi \hat{S}_{3}}$ at stage III. The braid operation with this type of coupling would yield a unitary operator of the same form found in Sec. VI.

The restriction of single-spin-species tunnelling can be met in the different realizations of the model analyzed above. First, consider the realization using a fractional quantum Hall liquid, in which an insulating trench separates two counterpropagating edge states [Fig. 1(b)]. In this realization, the labels "spin up" and "spin down" indicate whether the quasiparticles are on the inner or outer edge, respectively. When we deform the system in order to put interfaces in proximity, we must specify whether this deformation shrinks the inner or the outer droplet of the quantum Hall liquid. Suppose we shrink the inner droplet.
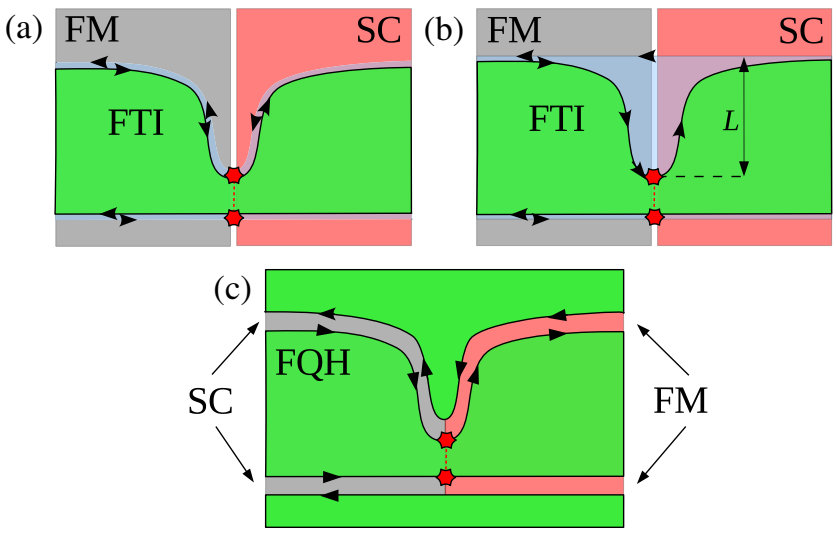

FIG. 6. Constrictions in the FTI and FQH realizations. We view the FTI as a combination of two FQH droplets with opposite spins and opposite filling fractions. The arrows show the direction of propagation of the edge states of the two FQH droplets. The red stars and lines indicate quasiparticle tunneling between the SC-FM interfaces on the edges. (a) Constriction in a FTI realization created by a gate potential, which acts on both spin species. In this case, quasiparticles of both spin species can tunnel across the constriction. (b) In a FTI realization, applying a local Zeeman field splits the spin-up and spin-down edge states, such that only one spin species can tunnel across the constriction. (c) In the FQH realization, "spin up" and "spin down" indicate whether the quasiparticles are on the edge of the inner or outer droplet, respectively. (Both droplets have the same filling fraction.) Since a constriction is achieved by shrinking either the inner or the outer droplet, only one quasiparticle species can tunnel. 
Then quasiparticle tunnelling between interfaces proceeds through the inner droplet. Therefore, in this case, quasiparticles can only tunnel from the inner edge at one interface location to the inner edge at another, as depicted in Fig. 6(c). Only electron-tunneling processes are allowed between the interfaces on the outer edge. However, electron tunnelling from the outer and inner edge is equivalent, as these tunnelings are related by Cooper-pair tunnelling or "spin flip" operators. To conclude, in this realization, choosing whether to deform the inner or the outer quantum Hall droplet selects which spin species of quasiparticles are allowed to tunnel between interfaces.

Let us now consider the realization of the system on the edge of a fractional topological insulator. Suppose that one can apply either ordinary gate potentials or Zeeman fields in the $z$ direction (by coupling to a nearby ferromagnet polarized along $z$ ), which act as opposite gate potentials for the two spin species. Then there are two ways of coupling two interfaces, depicted in Fig. 6. One can either create a constriction in both spin species by applying an appropriate gate voltage [Fig. 6(a)], which allows quasiparticle tunnelling of both spin species between the interfaces across the constriction, or create a constriction for one spin species only, e.g., spin up [Fig. 6(b)], in which case only that spin species tunnels. Note that, in the latter case, we have split the spin-up and spin-down edge states into two counterpropagating edge modes, which become gapless. However, if the length of the split region is $L$, there still is a finite-size gap of the order of $v_{\mathrm{F}} / L$, where $v_{\mathrm{F}}$ is the Fermi velocity on the edge. The tunnelling of quasiparticles of spin up across the constriction, on the other hand, is enhanced by a factor of the order of $\exp (L / \xi)$ relative to that of spin down, where $\xi$ is the correlation length in the bulk. Therefore, the tunnelling of spin-up quasiparticles can, in principle, be enhanced parametrically without reducing the gap considerably.

\section{APPENDIX D: YANG-BAXTER EQUATIONS}

Here, we verify that the unitaries representing braiding of two neighboring interfaces by tunneling of quasiparticles satisfy the Yang-Baxter equations. Imagine that we start from three consecutive interfaces, 1,2 , and 3, shown in Fig. 3. The segment between 1 and 2 is a superconducting (SC) segment, and the segment between 2 and 3 is a ferromagnetic (FM) segment. $e^{i \pi \hat{Q}}$ and $e^{i \pi \hat{S}}$ are the charge and spin operators acting on the SC and FM segments, respectively. In terms of these operators, one can express the unitary matrices that correspond to braiding $(1,2)$ and $(2,3)$ :

$$
\begin{aligned}
& \hat{U}_{12}=e^{i(\pi m / 2) \hat{Q}^{2}}=\frac{1}{\sqrt{2 m}} \sum_{k=0}^{2 m-1} e^{-i(\pi / 2 m) k^{2}+i(\pi / 4)} e^{i \pi k \hat{Q}}, \\
& \hat{U}_{23}=e^{i(\pi m / 2) \hat{S}^{2}}=\frac{1}{\sqrt{2 m}} \sum_{k=0}^{2 m-1} e^{-i(\pi / 2 m) k^{2}+i(\pi / 4)} e^{i \pi k \hat{S}} .
\end{aligned}
$$

Here, we have used the expansion of the braiding matrices in terms of the spin and charge operators and their harmonics. As in Sec. VII, we have fixed the charge-independent (spin-independent) global phase factor to zero.

The Yang-Baxter equations state that

$$
\hat{U}_{12} \hat{U}_{23} \hat{U}_{12}=\hat{U}_{23} \hat{U}_{12} \hat{U}_{23} \text {. }
$$

This relation can be understood pictorially, as shown in Fig. 3. Inserting Eqs. (D1) into the left-hand side of (D2), and using $e^{i \pi \hat{Q}} e^{i \pi \hat{S}}=e^{-i(\pi / m)} e^{i \pi \hat{S}} e^{i \pi \hat{Q}}$, we obtain

$$
\begin{aligned}
\hat{U}_{12} \hat{U}_{23} \hat{U}_{12}= & \sum_{k_{1}, k_{2}, k_{3}} \frac{e^{-i(\pi / 2 m)\left(k_{1}^{2}+k_{2}^{2}+k_{3}^{2}\right)+i(3 \pi / 4)}}{(2 m)^{(3 / 2)}} \\
& \times e^{i \pi k_{1} \hat{Q}} e^{i \pi k_{2} \hat{S}} e^{i \pi k_{3} \hat{Q}} \\
= & \sum_{k_{1}, k_{2}, k_{3}} \frac{e^{-i(\pi / 2 m)\left[\left(k_{1}-k_{2}\right)^{2}+k_{3}^{2}\right]+i(3 \pi / 4)}}{(2 m)^{(3 / 2)}} \\
& \times e^{i \pi k_{2} \hat{S}} e^{i \pi\left(k_{1}+k_{3}\right) \hat{Q}}
\end{aligned}
$$

The sums over $k_{1,2,3}$ run from 0 to $2 m-1$. Changing variables $k_{2} \rightarrow k_{1}+k_{2}$, and $k_{3} \rightarrow k_{3}-k_{1}$,

$$
\begin{aligned}
\hat{U}_{12} \hat{U}_{23} \hat{U}_{12}= & \sum_{k_{1}, k_{2}, k_{3}} \frac{e^{-i(\pi / 2 m)\left[k_{2}^{2}+\left(k_{3}-k_{1}\right)^{2}\right]+i(3 \pi / 4)}}{(2 m)^{(3 / 2)}} \\
& \times e^{i \pi\left(k_{1}+k_{2}\right) \hat{S}} e^{i \pi k_{3} \hat{Q}} \\
= & \sum_{k_{1}, k_{2}, k_{3}} \frac{e^{-i(\pi / 2 m)\left(k_{1}^{2}+k_{2}^{2}+k_{3}^{2}\right)+i(3 \pi / 4)}}{(2 m)^{(3 / 2)}} \\
& \times e^{i \pi k_{2} \hat{S}} e^{i \pi k_{3} \hat{Q}} e^{i \pi k_{1} \hat{S}} \\
= & e^{i(\pi m / 2) \hat{S}^{2}} e^{i(\pi m / 2) \hat{Q}^{2}} e^{i(\pi m / 2) \hat{S}^{2}} \\
= & \hat{U}_{23} \hat{U}_{12} \hat{U}_{23} .
\end{aligned}
$$

In the third line, we have commuted $e^{i \pi k_{1} \hat{S}}$ with $e^{i \pi k_{3} \hat{Q}}$. This step establishes the Yang-Baxter equation for the braiding matrices (D1). Conjugating Eq. (D2) by $e^{i \pi \hat{Q}_{L} k_{1}} e^{-i \pi \hat{S}_{R} k_{2}}$, where $e^{i \pi \hat{Q}_{L}}$ correspond to the charge in the SC segment on the left of $e^{i \pi \hat{Q}}$, and $e^{i \pi \hat{S}_{R}}$ to the spin in the FM segment on the right of $e^{i \pi \hat{S}}$, we obtain Eq. (27).

\section{APPENDIX E: MORE ON THE BRAIDING AND TOPOLOGICAL SPIN OF BOUNDARY ANYONS}

In Sec. VII, we derived the representation of the braid group using an analogy to braiding properties of anyons in two dimensions. The derivation proceeded using two important assumptions, and below we explain why these assumptions actually follow from properties of twodimensional anyons.

\section{Properties of the particle exchanges}

Consider a two-dimensional theory in which particle of type $a$ is exchanged first with particle of type $b$ and then 
(i)

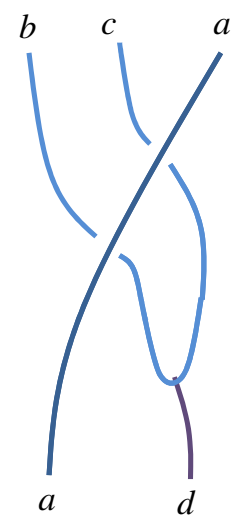

(ii)

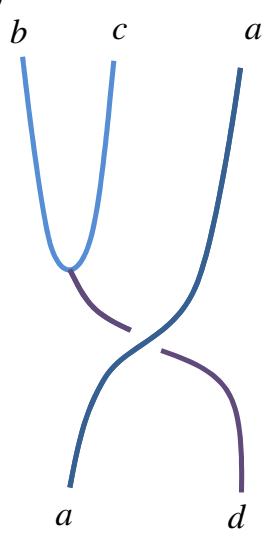

FIG. 7. Properties of particle exchanges. (i) Two consecutive exchanges, first between particles $a$ and $b$, and then between particles $a$ and $c$. (ii) Particle $a$ is exchanged with a particle $d$, which is the particle resulting from fusing $b$ and $c$. For twodimensional anyons, a proper definition of the braid matrix between any two particle types establishes an equality between (i) and (ii). For the case $a=b=c=X$ and $d=q$, we use only the property that the two braids in (i) move the label $q$ one segment to the left, as shown in (ii). For the case where $a=q_{1}$, $b=q_{2}, c=q_{3}$, and $d=q_{2}+q_{3} \bmod 2 m$, we show that the two sides are indeed equal.

with particle of type $c$. The operation should depend only on the type of particle $a$, and the total topological charge of particles $b$ and $c$. The operation should not be able to distinguish the finer splitting of the combined charge into the charges $b$ and $c$. This condition can be summarized pictorially in Fig. 7.

Importantly, the conditions summarized in Fig. 7 hold in our one-dimensional system, for the representation derived in Sec. VII, as we shall show below. The importance of these conditions to our derivation in Sec. VII is threefold, since it can be used to (a) show that the process in Fig. 4(c) indeed exchanges the charges of the two segments; (b) show that the TS of the $q_{1}, q_{2}$ composite is equal to the TS of $q_{1}+q_{2} \bmod 2 m$; (c) show that the Yang-Baxter equations hold for a braid of any two particle types. Therefore, the only assumption necessary for the derivation presented in Sec. VII is that the conditions summarized in Fig. 7 hold. For simplicity, we fix the $q$-independent phase of the braid matrix to zero.

In the following discussion, we shall study three important cases, for which we verify explicitly that the conditions in Fig. 7 hold in our one-dimensional system. These three cases are used to verify the assumptions (a) and (b) above.

$$
\text { a. } a=b=c=X, d=q
$$

For concreteness, consider four neighboring interfaces $X_{1}-X_{4}$, flanking alternating $\mathrm{FM}, \mathrm{SC}$, and FM segments. The operators corresponding to the charges in the different segments are $\chi_{3 \uparrow} \chi_{2 \uparrow}^{\dagger}=e^{i \pi \hat{Q}}, \chi_{2 \uparrow} \chi_{1 \uparrow}^{\dagger}=e^{i \pi \hat{S}_{1}}, \chi_{3 \uparrow} \chi_{4 \uparrow}^{\dagger}=e^{i \pi \hat{S}_{2}}$.

We shall also use $\chi_{4 \uparrow} \chi_{1 \uparrow}^{\dagger}=e^{i \pi \hat{S}_{1}} e^{i \pi \hat{Q}} e^{i \pi \hat{S}_{2}}$. Consider an initial state of the system which is an eigenstate of $e^{i \pi \hat{Q}}$ with eigenvalue $e^{i(\pi / m) q}$, and of $e^{i \pi \hat{S}_{1}+\hat{S}_{2}}$ with eigenvalue $e^{i(\pi / m) s_{\mathrm{tot}}}$

$$
\left|q, s_{\mathrm{tot}}\right\rangle=\frac{1}{\sqrt{2 m}} \sum_{n=0}^{2 m-1} e^{i(\pi / m) q n}\left|s_{1}=-n, s_{2}=s_{\mathrm{tot}}+n\right\rangle,
$$

where $s_{1}$ and $s_{2}$ correspond to $e^{i \pi \hat{S}_{1}}$ and $e^{i \pi \hat{S}_{2}}$. Note that such a state is also an eigenstate of $\chi_{4 \uparrow} \chi_{1 \uparrow}^{\dagger}$.

Following Fig. 7, drawing (i), consider two braid operations first between $X_{1}$ and $X_{2}$ and then between $X_{2}$ and $X_{3}$. Using $e^{i \pi \hat{Q}}\left|s_{1}, s_{2}\right\rangle=\left|s_{1}-1, s_{2}+1\right\rangle$ and the Fourier representation of the braid operators, the resulting state is

$$
\begin{aligned}
& (2 m) e^{-i(\pi / 2)} \hat{U}_{12}(\hat{Q}) \hat{U}_{23}\left(\hat{S}_{1}\right)\left|q, s_{\mathrm{tot}}\right\rangle \\
& \quad=\sum_{n, p, k=0}^{2 m-1} e^{i(\pi / 2 m)\left[-\left(p^{2}+k^{2}\right)+2 q n-2 n p\right]}\left|-n-k, s_{\mathrm{tot}}+n+k\right\rangle .
\end{aligned}
$$

Denoting $l=n+k$, we arrive at

$$
\begin{aligned}
& (2 m) e^{-i(\pi / 2)} \hat{U}_{12}(\hat{Q}) \hat{U}_{23}\left(\hat{S}_{1}\right)\left|q, s_{\mathrm{tot}}\right\rangle \\
& =\sum_{n, p, l=0}^{2 m-1} e^{i(\pi / 2 m)\left\{-\left[(p+n)^{2}+l^{2}\right]+2(q+l) n\right\}}\left|-l, s_{\mathrm{tot}}+l\right\rangle,
\end{aligned}
$$

and therefore

$$
\begin{aligned}
& (2 m) e^{-i(\pi / 2)} \hat{U}_{12}(\hat{Q}) \hat{U}_{23}\left(\hat{S}_{1}\right)\left|q, s_{\mathrm{tot}}\right\rangle \\
& \quad=\left(\sum_{r=0}^{2 m-1} e^{-i(\pi / 2 m) r^{2}}\right) e^{-i(\pi / 2 m) q^{2}}\left|s_{1}=q, s_{2}=s_{\mathrm{tot}}-q\right\rangle .
\end{aligned}
$$

We see, therefore, that the two consecutive exchanges are equivalent to moving the charge $q$ one segment to the left, i.e., $e^{i(\pi q / m)}$ becomes the eigenvalue of $\chi_{2 \uparrow} \chi_{1 \uparrow}^{\dagger}=e^{i \pi \hat{S}_{1}}$.

That result is exactly what is indicated in Fig. 7, drawing (ii). The state is also multiplied by a phase that depends on the gauge choices for the different basis [10]. Importantly, note that we could have chosen to fuse $X_{1}$ with a different interface $X_{i}$ (as long as it is not between 1 and 3), to form a charge $\chi_{i \uparrow} \chi_{1 \uparrow}^{\dagger}$, which would again commute with $\chi_{3 \uparrow} \chi_{2 \uparrow}^{\dagger}$. An analysis identical to the above would yield the same result, with $\chi_{i \uparrow}$ replacing $\chi_{4 \uparrow}$.

\section{b. Exchange of two q's}

Using the above, we would now like to show that the four exchanges depicted in Fig. 4(c) indeed correspond to exchanging two charges $q_{1}$ and $q_{2}$. Consider four neighboring interfaces $X_{0}-X_{3}$, flanking alternating $\mathrm{SC}$, FM, SC segments, where the initial state is $\left|q_{1}, q_{2}\right\rangle$, 
corresponding to an eigenstate of $\chi_{1 \uparrow} \chi_{0 \uparrow}^{\dagger}=e^{i \pi \hat{Q}_{1}}$ and $\chi_{3 \uparrow} \chi_{2 \uparrow}^{\dagger}=e^{i \pi \hat{Q}_{2}}$.

Indeed, using the above results, we see that performing the exchanges $X_{1}$ with $X_{2}$, and then $X_{2}$ and $X_{3}$, would result in an eigenstate of $\chi_{2 \uparrow} \chi_{1 \uparrow}^{\dagger}=e^{i \pi \hat{S}}$ with eigenvalue $e^{i(\pi / m) q_{2}}$. Now, performing the exchanges $X_{0}$ with $X_{1}$, and then $X_{1}$ and $X_{2}$, and again using the previous results, we see that the resulting state is an eigenstate of $\chi_{1 \uparrow} \chi_{0 \uparrow}^{\dagger}=e^{i \pi \hat{Q}_{1}}$ with eigenvalue $e^{i(\pi / m) q_{2}}$. Therefore, it is an eigenstate of $\chi_{3 \uparrow} \chi_{2 \uparrow}^{\dagger}=e^{i \pi \hat{Q}_{2}}$ with eigenvalue $e^{i(\pi / m) q_{1}}$. (The total charge in the two segments is preserved.) Therefore, the two charges have been exchanged by the sequence depicted in Fig. 4(c).

We shall now explicitly calculate the phase factor resulting from an exchange of two charges. Let us denote the operation at point by $U_{\hat{Q}_{1}, \hat{Q}_{2}}$. We would now like to verify that

$$
\begin{aligned}
\hat{U}_{\hat{Q}_{1}, \hat{Q}_{2}}\left|q_{1}, q_{2}\right\rangle & \equiv \hat{U}_{23}(\hat{S}) \hat{U}_{34}\left(\hat{Q}_{2}\right) \hat{U}_{12}\left(\hat{Q}_{1}\right) \hat{U}_{23}(\hat{S})\left|q_{1}, q_{2}\right\rangle \\
& =e^{i \phi\left(q_{1}, q_{2}\right)}\left|q_{2}, q_{1}\right\rangle
\end{aligned}
$$

and find the Abelian phase $\phi\left(q_{1}, q_{2}\right)$ associated with this exchange. The sequence of the four-braid operation in the above equation corresponds to Fig. 4(c). Using $e^{i \pi \hat{S}}\left|q_{1}, q_{2}\right\rangle=\left|q_{1}-1, q_{2}+1\right\rangle$ and the Fourier representation of the braid operators, we arrive at

$$
\sum_{n, p=0}^{2 m-1} e^{i(\pi / 2 m)\left[-\left(n^{2}+p^{2}\right)+\left(q_{1}-n\right)^{2}+\left(q_{2}+n\right)^{2}\right]}\left|q_{1}-n-p, q_{2}+n+p\right\rangle .
$$

Denoting $l=p+n$, we arrive at

$$
\sum_{n, l=0}^{2 m-1} e^{i(\pi / 2 m)\left[\left(-l^{2}+2 n l\right)+2\left(q_{2}-q_{1}\right) n+q_{1}^{2}+q_{2}^{2}\right]}\left|q_{1}-l, q_{2}+l\right\rangle .
$$

The sum over $n$ forces $l=q_{1}-q_{2}$, which therefore gives

$$
\hat{U}_{\hat{Q}_{1}, \hat{Q}_{2}}\left|q_{1}, q_{2}\right\rangle=e^{i(\pi / m) q_{1} q_{2}}\left|q_{2}, q_{1}\right\rangle .
$$

Therefore, Eq. (E5) holds, and the Abelian phase for interchanging two charges $q_{1}$ and $q_{2}$ is just $e^{i(\pi / m) q_{1} q_{2}}$, which is consistent with the topological spin for the charges, $\theta_{q}=e^{i(\pi / m) q^{2}}$, Eq. (29).

\section{c. $a=q_{1}, b=q_{2}, c=q_{3}, d=q_{2}+q_{3} \bmod 2 m$}

Consider three consecutive SC segments, and an initial state $\left|q_{1}, q_{2}, q_{3}\right\rangle$, corresponding to an eigenstate of $e^{i \pi \hat{Q}_{1}}$, $e^{i \pi \hat{Q}_{2}}$, and $e^{i \pi \hat{Q}_{3}}$. On the left side of Fig. 7, we first have an exchange of the charge of the first and second segments, resulting, according to the above discussion, in the state $e^{i(\pi / m) q_{1} q_{2}}\left|q_{2}, q_{1}, q_{3}\right\rangle$, followed by an exchange of the second and third segment, resulting in $e^{i(\pi / m)\left(q_{1} q_{2}+q_{1} q_{3}\right)}\left|q_{2}, q_{3}, q_{1}\right\rangle$. Clearly, that is exactly the result of the operation on the left side of Fig. 7, in which the total charge $q_{2}+q_{3}$ is exchanged with $q_{1}$ and then split again into the two segments.

\section{Consistency check}

In this section we want to verify that the topological spin of the interfaces is indeed well defined, i.e., that the phase acquired by the operation defining the topological spin is independent of the initial state of the system. Consider the procedure in Fig. 4(e). Interface 1 flanks the left side of a SC segment, which corresponds to $e^{i \pi \hat{Q}}$. The initial state can be taken as an eigenstate of $e^{i \pi \hat{Q}}$ with eigenvalue $q$. A FM segment is nucleated to the right of interface 1, separating the SC segment into two. The FM segment is nucleated with spin zero. Therefore, the state after the nucleation of this segment is

$|\psi(q, s=0)\rangle=\frac{1}{\sqrt{2 m}} \sum_{n=0}^{2 m-1}\left|q_{1}=q-n, q_{2}=n\right\rangle$.

As explained in Sec. VII, the braid operation between interfaces 1 and 2 does not change the charge of the SC segment between interfaces 1 and 2, the charge between 3 and 4 , and therefore the total charge $q$. (Numbering increases to the right.) Therefore,

$\hat{U}_{12}|\psi(q, s)\rangle=\frac{1}{\sqrt{2 m}} \sum_{n=0}^{2 m-1} e^{i \varphi(q-n)}\left|q_{1}=q-n, q_{2}=n\right\rangle$,

where we keep the phase function $\varphi(q)$ completely general. We now project on the subspace with $e^{i \pi \hat{S}}=1$ in the FM segment, i.e., we apply the projector

$$
\Pi_{s=0}=\sum_{q}|\psi(q, s=0)\rangle\langle\psi(s=0, q)|,
$$

where the identity operation is implicitly assumed to act on all other degrees of freedom. Applying the projection yields (up to normalization),

$$
\theta_{X}=\sum_{n=0}^{2 m-1} e^{i \varphi(q-n)}
$$

Importantly, $\theta_{X}$ does not depend on $q$, as the sum runs over all possible values for charges.

[1] J. M. Leinaas and J. Myrheim, On the Theory of Identical Particles, Nuovo Cimento Soc. Ital. Fis. B 37, 1 (1977).

[2] Frank Wilczek, Quantum Mechanics of Fractional-Spin Particles, Phys. Rev. Lett. 49, 957 (1982).

[3] B. Blok and X.-G. Wen, Many-Body Systems with NonAbelian Statistics, Nucl. Phys. B374, 615 (1992). 
[4] Ady Stern, Non-Abelian States of Matter, Nature (London) 464, 187 (2010).

[5] Gregory Moore and Nicholas Read, Nonabelions in the Fractional Quantum Hall Effect, Nucl. Phys. B360, 362 (1991).

[6] Daniel Arovas, J.R. Schrieffer, and Frank Wilczek, Fractional Statistics and the Quantum Hall Effect, Phys. Rev. Lett. 53, 722 (1984).

[7] Alexei Yu. Kitaev, Fault-Tolerant Quantum Computation by Anyons, Ann. Phys. (N.Y.) 303, 2 (2003).

[8] N. Read and E. Rezayi, Quasiholes and Fermionic Zero Modes of Paired Fractional Quantum Hall States: The Mechanism for Non-Abelian Statistics, Phys. Rev. B 54, 16864 (1996).

[9] Ady Stern, Anyons and the Quantum Hall Effect: A Pedagogical Review Ann. Phys. (N.Y.) 323, 204 (2008).

[10] Alexei Kitaev, Anyons in an Exactly Solved Model and Beyond, Ann. Phys. (N.Y.) 321, 2 (2006).

[11] N. Read and Dmitry Green, Paired States of Fermions in Two Dimensions with Breaking of Parity and TimeReversal Symmetries and the Fractional Quantum Hall Effect, Phys. Rev. B 61, 10267 (2000).

[12] D. A. Ivanov, Non-Abelian Statistics of Half-Quantum Vortices in p-Wave Superconductors, Phys. Rev. Lett. 86, 268 (2001).

[13] C. Nayak and F. Wilczek, 2n-Quasihole States Realize $2^{n-1}$-Dimensional Spinor Braiding Statistics in Paired Quantum Hall States, Nucl. Phys. B479, 529 (1996).

[14] Liang Fu and C.L. Kane, Superconducting Proximity Effect and Majorana Fermions at the Surface of a Topological Insulator, Phys. Rev. Lett. 100, 096407 (2008).

[15] Liang $\mathrm{Fu}$ and C.L. Kane, Josephson Current and Noise at a Superconductor/Quantum-Spin-Hall-Insulator/ Superconductor Junction, Phys. Rev. B 79, 161408 (2009).

[16] Jay D. Sau, Roman M. Lutchyn, Sumanta Tewari, and Sankar Das Sarma, Generic New Platform for Topological Quantum Computation Using Semiconductor Heterostructures, Phys. Rev. Lett. 104, 040502 (2010).

[17] Xiao-Liang Qi, Taylor L. Hughes, and Shou-Cheng Zhang, Chiral Topological Superconductor from the Quantum Hall State, Phys. Rev. B 82, 184516 (2010).

[18] Jay D. Sau, Sumanta Tewari, Roman M. Lutchyn, Tudor D. Stanescu, and S. Das Sarma, Non-Abelian Quantum Order in Spin-Orbit-Coupled Semiconductors: Search for Topological Majorana Particles in Solid-State Systems, Phys. Rev. B 82, 214509 (2010).

[19] Tudor D. Stanescu, Jay D. Sau, Roman M. Lutchyn, and S. Das Sarma, Proximity Effect at the SuperconductorTopological Insulator Interface, Phys. Rev. B 81, 241310 (2010).

[20] Yuval Oreg, Gil Refael, and Felix von Oppen, Helical Liquids and Majorana Bound States in Quantum Wires, Phys. Rev. Lett. 105, 177002 (2010).

[21] Roman M. Lutchyn, Jay D. Sau, and S. Das Sarma, Majorana Fermions and a Topological Phase Transition in Semiconductor-Superconductor Heterostructures, Phys. Rev. Lett. 105, 077001 (2010).

[22] A. Cook and M. Franz, Majorana Fermions in a Topological-Insulator Nanowire Proximity-Coupled to an s-Wave Superconductor, Phys. Rev. B 84, 201105 (2011).
[23] V. Mourik, K. Zuo, S. M. Frolov, S. R. Plissard, E. P. A. M. Bakkers, and L. P. Kouwenhoven, Signatures of Majorana Fermions in Hybrid Superconductor-Semiconductor Nanowire Devices, Science, 336, 1003 (2012).

[24] R. L. Willett, L. N. Pfeiffer, and K. W. West, Measurement of Filling Factor 5/2 Quasiparticle Interference with Observation of Charge e/4 and e/2 Period Oscillations Proc. Natl. Acad. Sci. U.S.A. 106, 8853 (2009).

[25] S. An, P. Jiang, H. Choi, W. Kang, S. H. Simon, L. N. Pfeiffer, K.W. West, and K.W. Baldwin, Braiding of Abelian and Non-Abelian Anyons in the Fractional Quantum Hall Effect, arXiv:1112.3400.

[26] L. P. Rokhinson, X. Liu, and J. K. Furdyna, Observation of the Fractional ac Josephson Effect: The Signature of Majorana Particles, arXiv:1204.4212.

[27] A. Das, Y. Ronen, Y. Most, Y. Oreg, M. Heiblum, and H Shtrikman, Evidence of Majorana Fermions in an Al-InAs Nanowire Topological Superconductor, arXiv:1205.7073.

[28] J. Alicea, New Directions in the Pursuit of Majorana Fermions in Solid State Systems, Rep. Prog. Phys. 75, 076501 (2012).

[29] C. W. J. Beenakker, Search for Majorana Fermions in Superconductors, arXiv:1112.1950.

[30] Chetan Nayak, Steven H. Simon, Ady Stern, Michael Freedman, and Sankar Das Sarma, Non-Abelian Anyons and Topological Quantum Computation, Rev. Mod. Phys. 80, 1083 (2008), and references therein.

[31] M. H. Freedman, Michael J. Larsen, and Zhenghan Wang, A Modular Functor Which Is Universal for Quantum Computation, Commun. Math. Phys. 227, 605 (2002).

[32] Michael Levin and Ady Stern, Fractional Topological Insulators, Phys. Rev. Lett. 103, 196803 (2009).

[33] C. L. Kane and E. J. Mele, $Z_{2}$ Topological Order and the Quantum Spin Hall Effect, Phys. Rev. Lett. 95, 146802 (2005).

[34] B. Andrei Bernevig and Shou-Cheng Zhang, Quantum Spin Hall Effect, Phys. Rev. Lett. 96, 106802 (2006).

[35] R. B. Laughlin, Anomalous Quantum Hall Effect: An Incompressible Quantum Fluid with Fractionally Charged Excitations, Phys. Rev. Lett. 50, 1395 (1983).

[36] Ady Stern, Felix von Oppen, and Eros Mariani, Geometric Phases and Quantum Entanglement as Building Blocks for Non-Abelian Quasiparticle Statistics, Phys. Rev. B 70, 205338 (2004).

[37] Jeffrey C. Y. Teo and C. L. Kane, Majorana Fermions and Non-Abelian Statistics in Three Dimensions, Phys. Rev. Lett. 104, 046401 (2010).

[38] Jason Alicea, Yuval Oreg, Gil Refael, Felix von Oppen, and Matthew P.A. Fisher, Non-Abelian Statistics and Topological Quantum Information Processing in 1d Wire Networks, Nature Phys. 7, 412 (2011).

[39] Jay D. Sau, David J. Clarke, and Sumanta Tewari, Controlling Non-Abelian Statistics of Majorana Fermions in Semiconductor Nanowires, Phys. Rev. B 84, 094505 (2011).

[40] Bertrand I. Halperin, Yuval Oreg, Ady Stern, Gil Refael, Jason Alicea, and Felix von Oppen, Adiabatic Manipulations of Majorana Fermions in a ThreeDimensional Network of Quantum Wires, Phys. Rev. B 85, 144501 (2012). 
[41] X.G. Wen, Chiral Luttinger Liquid and the Edge Excitations in the Fractional Quantum Hall States, Phys. Rev. B 41, 12838 (1990).

[42] Dung-Hai Lee and Xiao-Gang Wen, Edge Excitations in the Fractional-Quantum-Hall Liquids, Phys. Rev. Lett. 66, 1765 (1991).

[43] H. Bombin, Topological Order with a Twist: Ising Anyons from an Abelian Model, Phys. Rev. Lett. 105, 030403 (2010).

[44] Maissam Barkeshli and Xiao-Liang Qi, Topological Nematic States and Non-Abelian Lattice Dislocations, Phys. Rev. X 2, 031013 (2012).

[45] Yi-Zhuang You and Xiao-Gang Wen, Projective NonAbelian Statistics of Dislocation Defects in a $\mathrm{ZN}$ Rotor Model, arXiv:1204.0113.

[46] Paul Fendley, Edge Modes, Zero Modes and Conserved Charges in Parafermion Chains, http://pirsa.org/ 11080023/; Parafermionic Edge Zero Modes in $\mathrm{Z}_{\mathrm{n}}$-Invariant Spin Chains, arXiv:1209.0472.

[47] E. Fradkin and L. P. Kadanoff, Disorder Variables and Parafermions in Two-Dimensional Statistical Mechanics, Nucl. Phys. B170, 1 (1980).

[48] This global phase in this identity can be proved using a Gauss sum. See, e.g., B. C. Berndt and R. J. Evans, The Determination of Gauss Sums, Bull. Am. Math. Soc. 5, 107 (1981).

[49] As noted in the previous section, the actual unitary evolution operators form a projective representation of the braid group, due to the path-dependent global phase factor. For simplicity of the presentation, in the following discussion, we shall assume that this global phase is path independent, allowing us to obtain the braid matrix up to an overall phase.

[50] J. Preskill, Topological Quantum Computation, California Institute of Technology 2004 (http://www.lorentz .leidenuniv.nl/quantumcomputers/literature/preskill_9.pdf).

[51] E. Rowell, R. Stong, and Zhenghan Wang, On Classification of Modular Tensor Categories, Commun. Math. Phys. 292, 343 (2009).

[52] Michael Freedman, Chetan Nayak, and Kevin Walker, Towards Universal Topological Quantum Computation in the $\nu=5 / 2$ Fractional Quantum Hall State, Phys. Rev. B 73, 245307 (2006).

[53] E. Berg, N.H. Lindner, G. Refael, and A. Stern (unpublished).

[54] F. Hassler, A. R. Akhmerov, C-Y. Hou, and C. W. J. Beenakker, Anyonic Interferometry without Anyons:
How a Flux Qubit Can Read Out a Topological Qubit, New J. Phys. 12, 125002 (2010).

[55] Liang Jiang, David Pekker, Jason Alicea, Gil Refael, Yuval Oreg, and Felix von Oppen, Unconventional Josephson Signatures of Majorana Bound States, Phys. Rev. Lett. 107, 236401 (2011).

[56] Lukasz Fidkowski and Alexei Kitaev, Effects of Interactions on the Topological Classification of Free Fermion Systems, Phys. Rev. B 81, 134509 (2010).

[57] Lukasz Fidkowski and Alexei Kitaev, Topological Phases of Fermions in One Dimension, Phys. Rev. B 83, 075103 (2011).

[58] Ari M. Turner, Frank Pollmann, and Erez Berg, Topological Phases of One-Dimensional Fermions: An Entanglement Point of View, Phys. Rev. B 83, 075102 (2011).

[59] Xie Chen, Zheng-Cheng Gu, and Xiao-Gang Wen, Complete Classification of One-Dimensional Gapped Quantum Phases in Interacting Spin Systems, Phys. Rev. B 84, 235128 (2011).

[60] Norbert Schuch, David Perez-Garcia, and Ignacio Cirac, Classifying Quantum Phases Using Matrix Product States and Projected Entangled Pair States, Phys. Rev. B 84, 165139 (2011).

[61] B.I. Halperin, Statistics of Quasiparticles and the Hierarchy of Fractional Quantized Hall States, Phys. Rev. Lett. 52, 1583 (1984).

[62] Michael Levin, F. J. Burnell, Maciej Koch-Janusz, and Ady Stern, Exactly Soluble Models for Fractional Topological Insulators in Two and Three Dimensions, Phys. Rev. B 84, 235145 (2011).

[63] B. Swingle, M. Barkeshli, J. McGreevy, and T. Senthil, Correlated Topological Insulators and the Fractional Magnetoelectric Effect, Phys. Rev. B 83, 195139 (2011).

[64] Joseph Maciejko, Xiao-Liang Qi, Andreas Karch, and Shou-Cheng Zhang, Fractional Topological Insulators in Three Dimensions, Phys. Rev. Lett. 105, 246809 (2010).

[65] D. J. Clarke, J. Alicea, and K. Shtengel, Exotic NonAbelian Anyons from Conventional Fractional Quantum Hall States, arXiv:1204.5479.

[66] M. Cheng, Superconducting Proximity Effect on the Edge of Fractional Topological Insulators, arXiv:1204.6084 [Phys. Rev. B (to be published)].

[67] A. Vaezi, Fractional Topological Superconductor with Fractionalized Majorana Fermions, arXiv:1204.6245. 